Supporting Information

\title{
Earth-Abundant Bimetallic Nanoparticle Catalysts for Aerobic Ammoxidation of Alcohols to Nitriles
}

Tomohiro Yasukawa, Xi Yang, and Shū Kobayashi* 


\section{Contents}

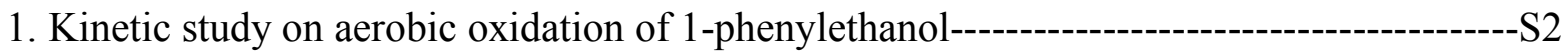

2. Additional optimization data-------------------------------------------------------------------S4

3. Studies of $\mathrm{NCI}-\mathrm{Co} / \mathrm{Cu}$ catalyzed aerobic oxidative esterification of alcohols for comparison---------------------------------------------------------------------------------------S4

4. STEM analysis and EDS mapping------------------------------------------------------------S5

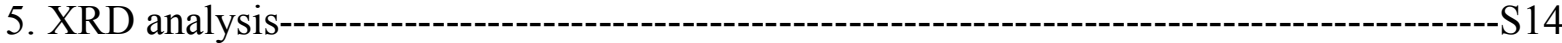

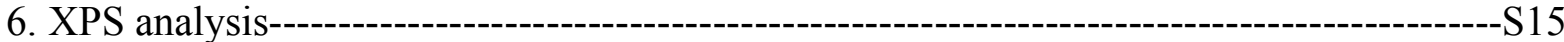

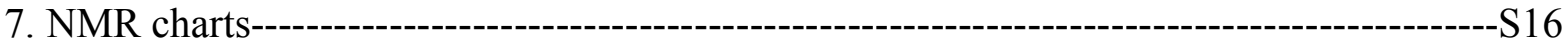

1. Kinetic study on aerobic oxidation of 1-phenylethanol

1-1. A typical procedure of secondary alcohol oxidation reaction profile: $\mathrm{NCI}-\mathrm{M}_{1} /\left(\mathrm{M}_{2}\right)(1$ mol\%) was added in a Carousel ${ }^{\mathrm{TM}}$ tube and dried with heat gun in vacuo. 1-Phenylethanol $(30.5 \mathrm{mg}, 0.25 \mathrm{mmol})$, anisole $(25-30 \mathrm{mg})$ and methanol $(2 \mathrm{~mL})$ was added to the Carousel tube under Ar atmosphere. The mixture was stirred under 1 bar of $\mathrm{O}_{2}$ atmosphere at $70{ }^{\circ} \mathrm{C}$. At each time, an aliquot of the reaction mixture $(\sim 0.02 \mathrm{~mL})$ was filtered through a silica gel packed disposable Pasteur pipette and washed with ethyl acetate to inject the GC analysis. The conversion of starting material and the yield of acetophenone were plotted against the reaction time. The natural logarithm of concentration of the starting material was plotted against the reaction time. The rate constant was determined by linear regression.

\section{1-2. Reaction profiles of secondary alcohol oxidation with different catalysts}
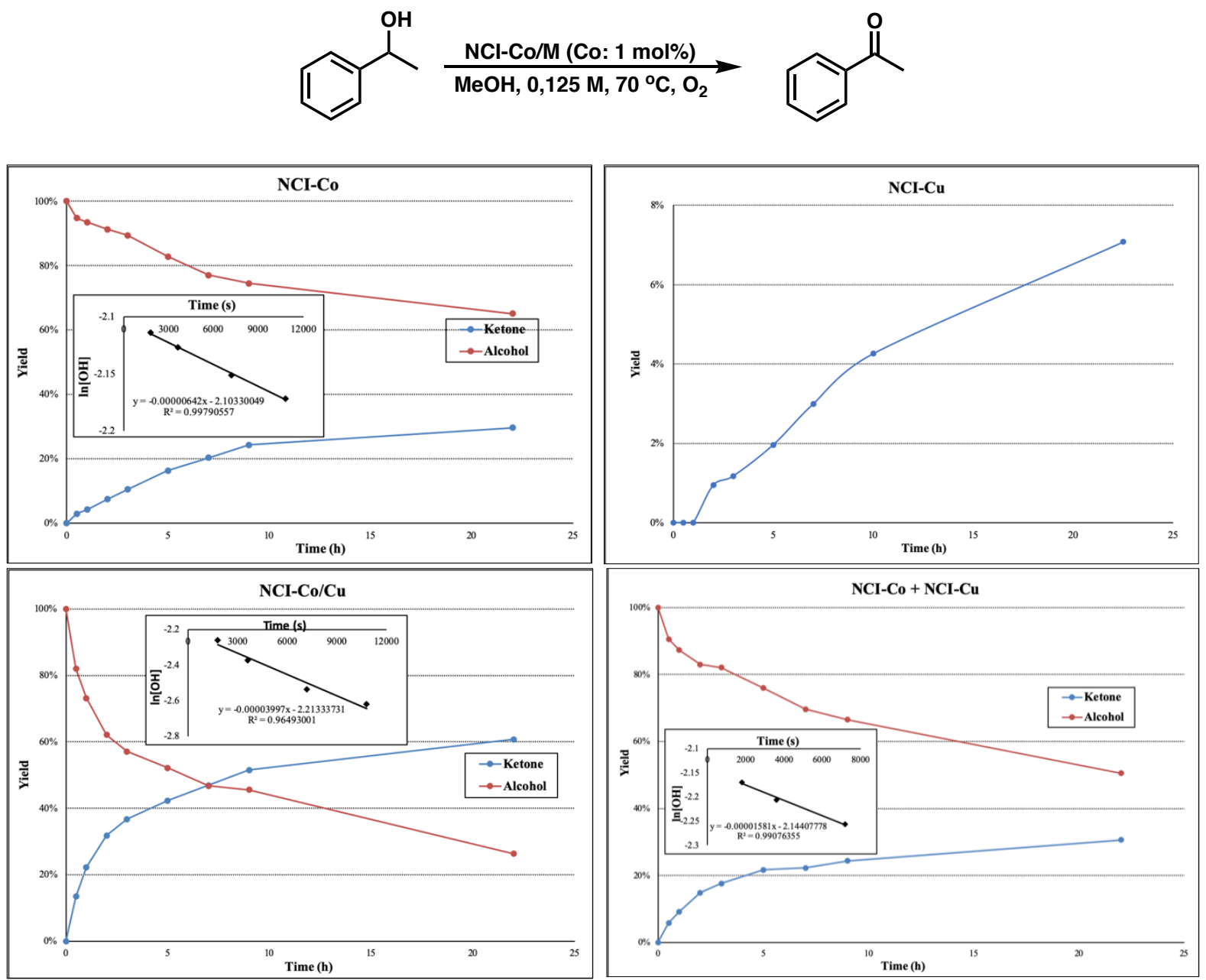
Figure S1. Reaction profiles in oxidation of secondary alcohols

Table S1. The reaction rate in oxidation of secondary alcohol

\begin{tabular}{cccc}
\hline Entry & Catalyst & Rate Constant $\left(\mathrm{s}^{-1}\right)$ & TOF $\left(\mathrm{h}^{-1}\right)^{\mathrm{a}}$ \\
\hline 1 & NCI-Co 1 & $6.4 \times 10^{-6}$ & 4.24 \\
$2^{\mathrm{b}}$ & NCI-Co 1 + NCI-Cu 2 & $1.6 \times 10^{-5}$ & 9.21 \\
3 & NCI-Co/Cu 4 & $4.0 \times 10^{-4}$ & 22.2 \\
4 & NCI-Cu 2 & $1.1 \times 10^{-6}$ & - \\
\hline
\end{tabular}

${ }^{\mathrm{a}}$ TOF of the initial hour. ${ }^{\mathrm{b}} 1 \mathrm{~mol} \%$ of NCI-Co 1 and $1 \mathrm{~mol} \%$ of NCI-Cu 2 were used.

Kinetic studies with aerobic oxidation of secondary alcohols indicated that $\mathrm{Cu}$ could facilitate in accelerating in oxidation from alcohols to carbonyl compounds, whereas NCI-Cu 2 monometallic catalyst alone showed significantly low activity. TOF of bimetallic NCI$\mathrm{Co} / \mathrm{Cu} 4$ catalyst had the best catalytic activity, which was significantly higher than that of monometallic $\mathrm{Co}$ and $\mathrm{Cu}$ catalysts combined, indicating the advantage of bimetallic formation. 


\section{Additional optimization data}

Table S2. Optimization of nitrile synthesis

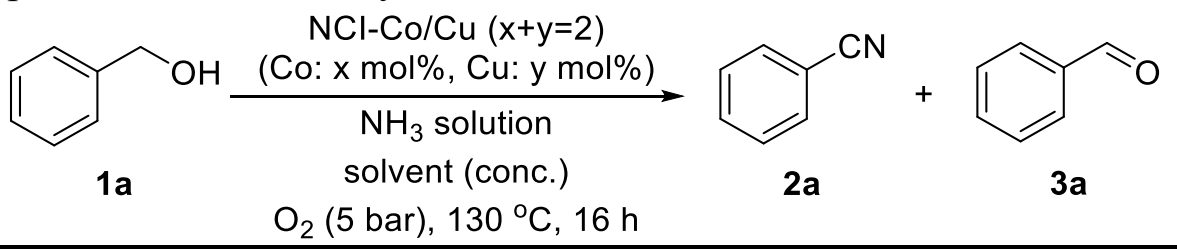

\begin{tabular}{|c|c|c|c|c|c|}
\hline Entry & $\mathrm{x} / \mathrm{y}$ & $\mathrm{NH}_{3}$ source & Solvent (Conc.) & $\begin{array}{l}\text { Yield } \\
(\%)\end{array}$ & $\begin{array}{c}3 \\
(\%)\end{array}$ \\
\hline 1 & $1.0 / 0$ & \multirow{4}{*}{$28 \%$ aq. $\mathrm{NH}_{3}$ (3.0 eq.) } & \multirow{4}{*}{$t \mathrm{AmOH}(0.125 \mathrm{M})$} & 3 & 6 \\
\hline 2 & $0 / 1.0$ & & & 11 & 2 \\
\hline 3 & $1.0 / 1.0$ & & & 29 & 7 \\
\hline 4 & $0.4 / 1.6$ & & & 41 & 6 \\
\hline & \multirow{2}{*}{$1.0 / 1.0$} & $2 \mathrm{M} \mathrm{NH}_{3}$ in $\mathrm{EtOH}(1.0 \mathrm{eq})$ & \multirow{2}{*}{$t \mathrm{AmOH}(0.25 \mathrm{M})$} & 45 & 11 \\
\hline 6 & & $2 \mathrm{M} \mathrm{NH}_{3}$ in $\mathrm{EtOH}(2.0 \mathrm{eq})$ & & 42 & 4 \\
\hline 7 & \multirow{2}{*}{$0.4 / 1.6$} & \multirow{2}{*}{$28 \%$ aq. $\mathrm{NH}_{3}$ (3.0 eq.) } & $t \mathrm{AmOH}(0.25 \mathrm{M})$ & 59 & 5 \\
\hline 8 & & & $\operatorname{MeCN}(0.25 \mathrm{M})$ & 33 & 12 \\
\hline 9 & \multirow{4}{*}{$0.4 / 1.6$} & $2 \mathrm{M} \mathrm{NH}_{3}$ in $\mathrm{EtOH}(1.0 \mathrm{eq})$ & \multirow{4}{*}{$t \mathrm{AmOH}(0.25 \mathrm{M})$} & 42 & 21 \\
\hline 10 & & $2 \mathrm{M} \mathrm{NH}_{3}$ in $\mathrm{EtOH}(2.0 \mathrm{eq})$ & & 42 & 6 \\
\hline 11 & & $2 \mathrm{M} \mathrm{NH}_{3}$ in $\mathrm{MeOH}(1.0 \mathrm{eq})$ & & 42 & 21 \\
\hline 12 & & $2 \mathrm{M} \mathrm{NH}_{3}$ in $i \mathrm{PrOH}(1.0$ eq $)$ & & 51 & 2 \\
\hline 13 & \multirow{5}{*}{$0.4 / 1.6$} & $28 \%$ aq. $\mathrm{NH}_{3}$ (3.0 eq.) & \multirow{5}{*}{$t \mathrm{AmOH}(0.5 \mathrm{M})$} & 79 & 2 \\
\hline 14 & & $28 \%$ aq. $\mathrm{NH}_{3}(2.0$ eq. $)$ & & 73 & 4 \\
\hline 15 & & $28 \%$ aq. $\mathrm{NH}_{3}(4.0$ eq. $)$ & & 73 & 1 \\
\hline 16 & & $28 \%$ aq. $\mathrm{NH}_{3}(1.5$ eq. $)$ & & 52 & 31 \\
\hline $17^{\mathrm{a}}$ & & $28 \%$ aq. $\mathrm{NH}_{3}$ (3.0 eq.) & & 96 & 1 \\
\hline
\end{tabular}

a $24 \mathrm{~h}$.

3. Studies of NCI-Co/Cu catalyzed aerobic oxidative esterification of alcohols for comparison

Following NCI-Fe-Cu 11 and NCI-Cu-Fe 12, we prepared $\mathrm{Co} / \mathrm{Cu}$ bimetallic catalysts where two metal salts were separately incorporated and sequentially reduced with $\mathrm{NaBH}_{4}$ (See Scheme 2 in the main text). In contrast to NCI-Fe/Cu catalyzed nitrile synthesis, comparable activities of both NCI-Co-Cu 13 and NCI-Cu-Co 14 with NCI-Co/Cu 4 were observed (Table S3). 
Table S3. Examination of separate NCI-Co/Cu NPs in aerobic oxidative esterification

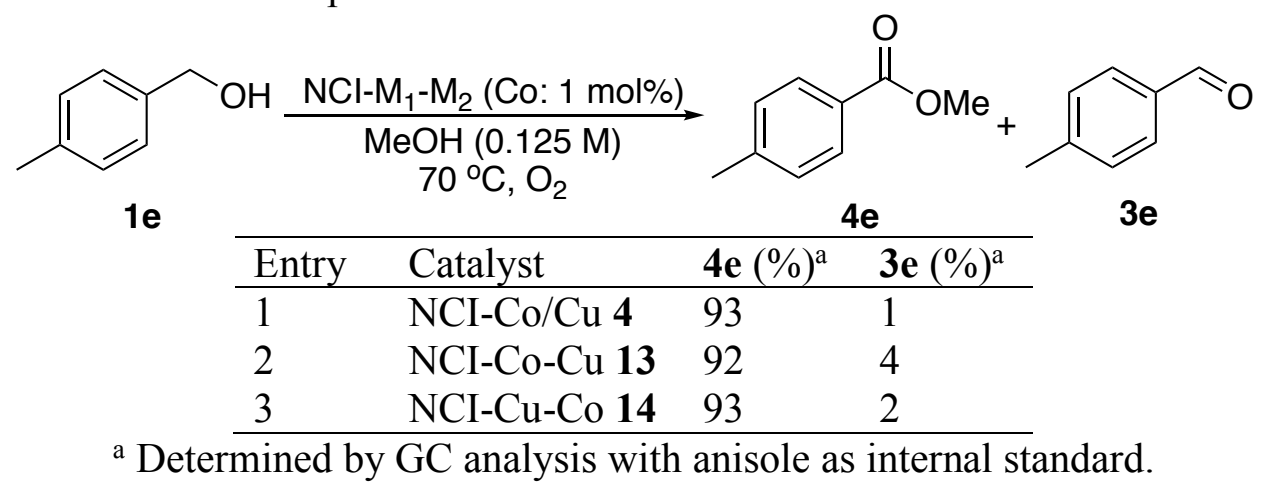

Acid leaching test was performed in $\mathrm{NCI}-\mathrm{Co} / \mathrm{Cu} 4$ catalyzed aerobic esterification of alcohols (Table S4). Similar to NCI-Fe/Cu, most of the $\mathrm{Cu}$ species were removed after acidwashing and comparable activity was observed between NCI-Co/Cu 4-H, NCI-Co 1, and NCI-Co 1-H.

These results indicated that the Co species that were removed by acid-washing were mostly inactive. STEM analysis confirmed that the size distribution of NPs was dramatically changed from $\sim 20 \mathrm{~nm}$ in NCI-Co/Cu 4 (see reference 3 in the main text) to $\sim 6 \mathrm{~nm}$ in NCI$\mathrm{Co} / \mathrm{Cu}$ 4-H (Figures S9 and S12) and EDS analysis of NCI-Co/Cu 4-H detected metal species in some areas where no visible particles were observed (Figure S9). XPS analysis of NCI$\mathrm{Co} / \mathrm{Cu}$ 4-H showed that although only very weak intensity of Co peak was observed, it seemed that no dramatic change of the oxidation state on the catalyst surface occurred (Figure S22 and reference 3 in the main text). On the other hands, no visible peak derived from $\mathrm{Cu}$ was observed in this analysis.

Table S4. Examination of acid-washed NCI-Co/Cu in aerobic oxidative esterification

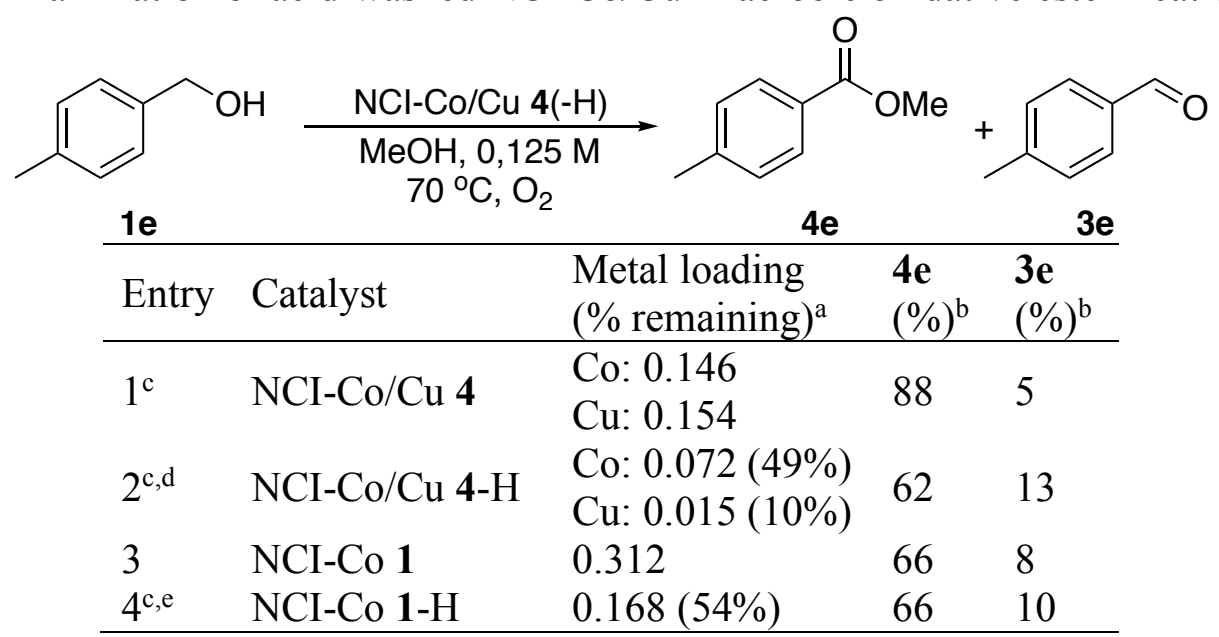

a Determined by ICP analysis $(\mathrm{mmol} / \mathrm{g})$. The value in parentheses indicates the ratio of the remaining metal to the loading of metal in the catalyst before washing. ${ }^{b}$ Determined by GC analysis with anisole as internal standard. ${ }^{c}$ Average of two different batches of catalysts. ${ }^{d}$ Same weight of catalyst was incorporated as that in entry $1 .{ }^{e}$ Same weight of catalyst was incorporated as that in entry 3. 
4. STEM analysis and EDS mapping
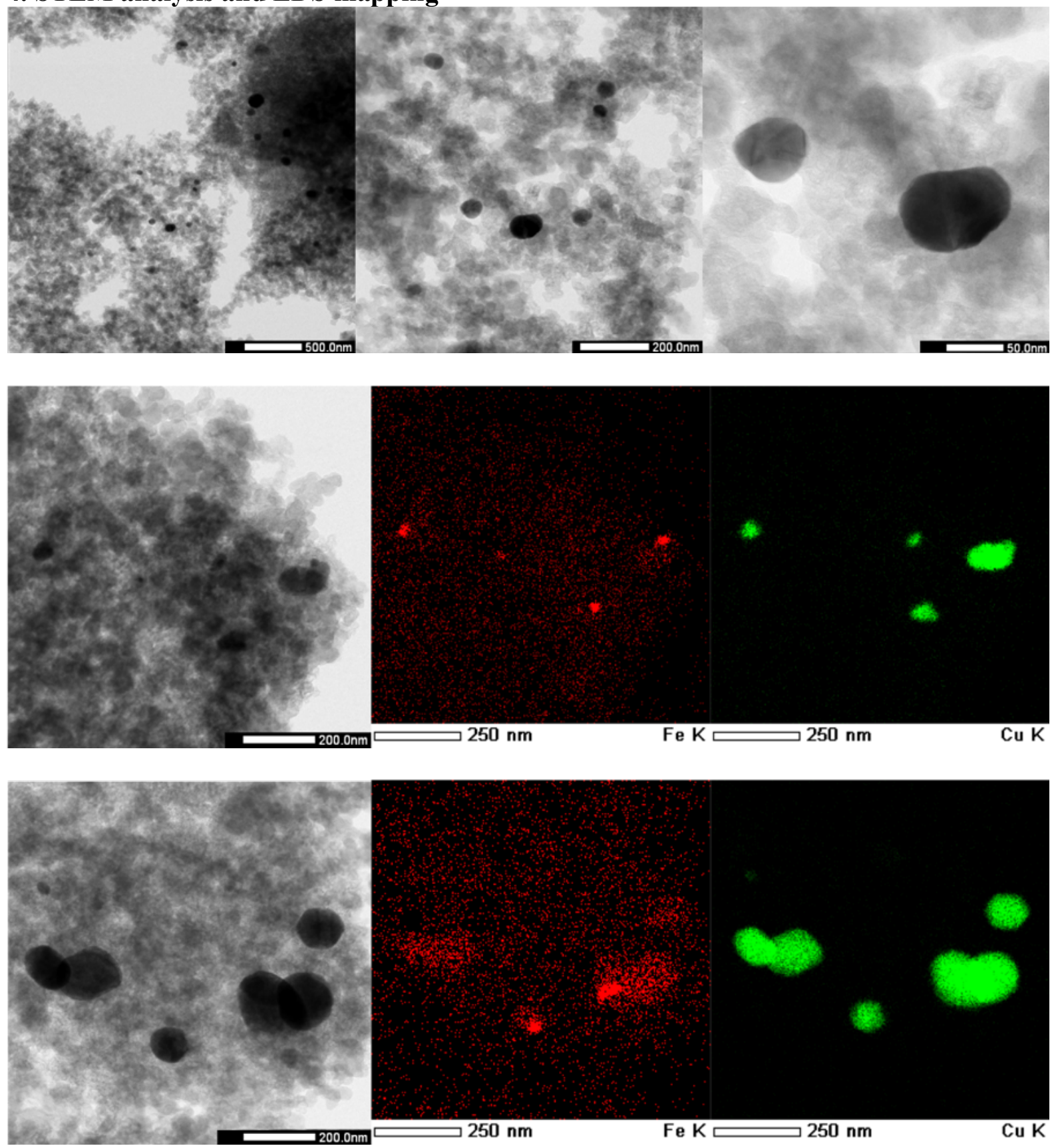

Figure S2. STEM images and EDS mapping of NCI-Fe/Cu 9 

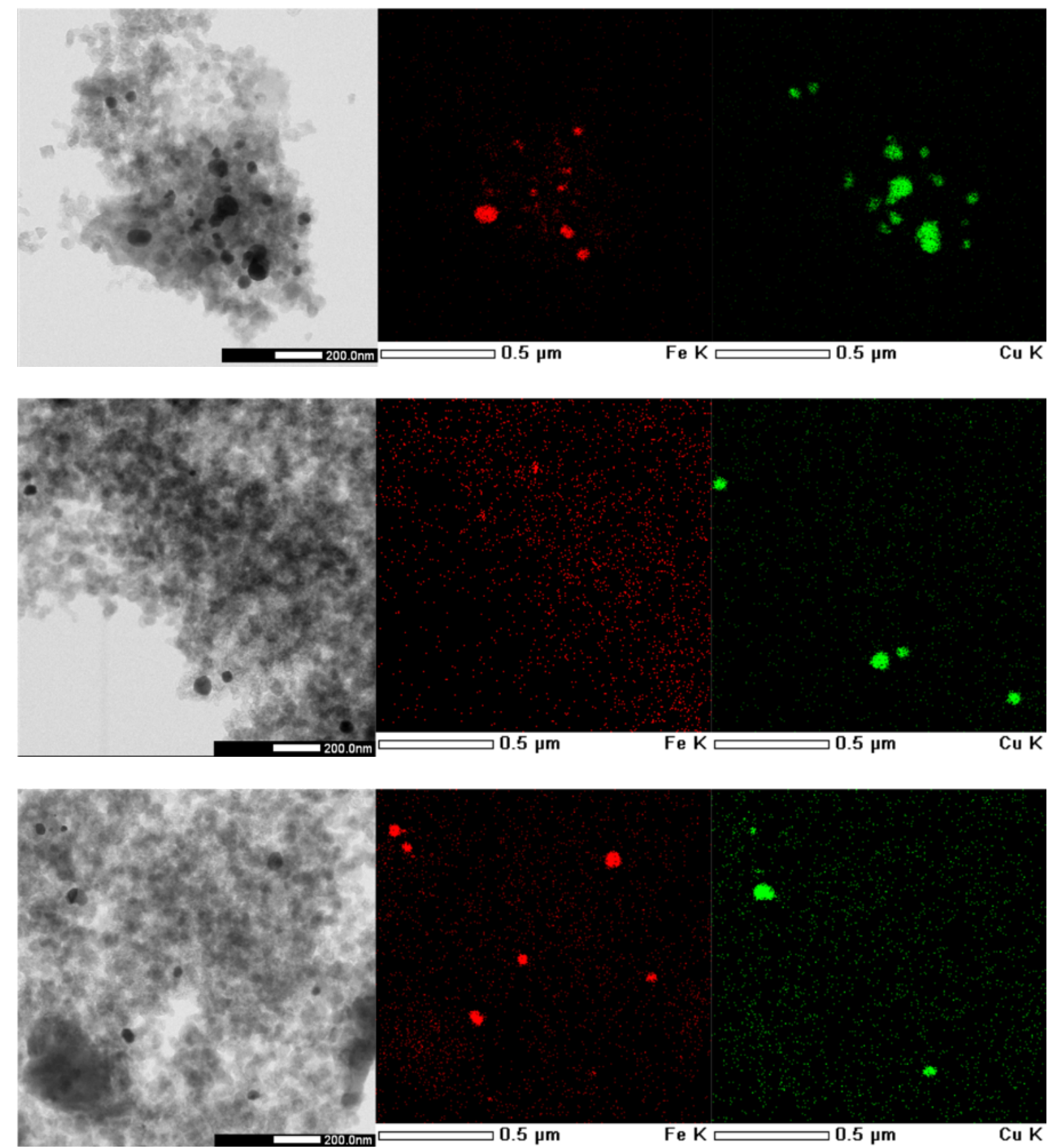

Figure S3. STEM images and EDS mapping of NCI-Fe-Cu 11 


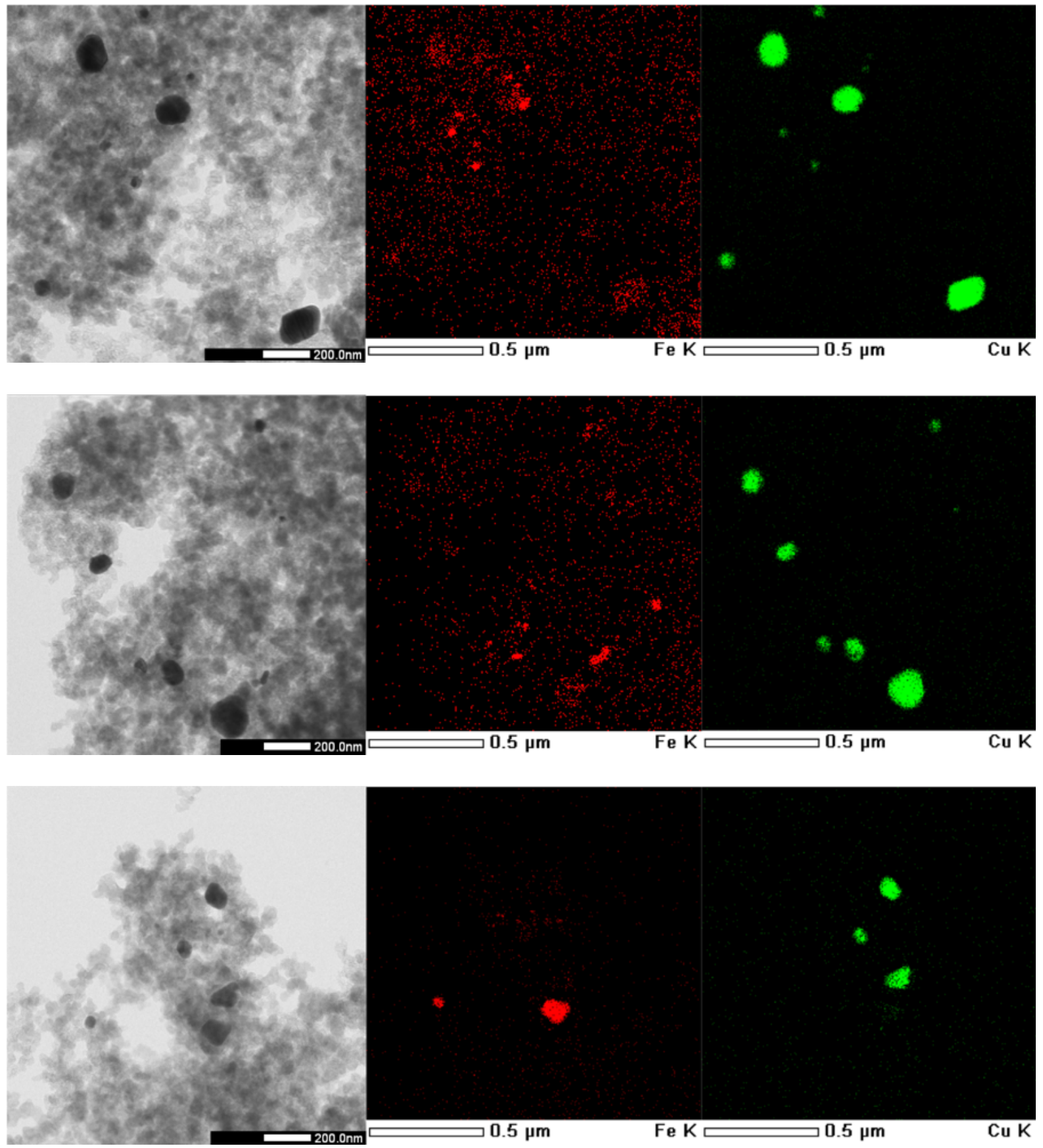

Figure S4. STEM images and EDS mapping of NCI-Cu-Fe 12 


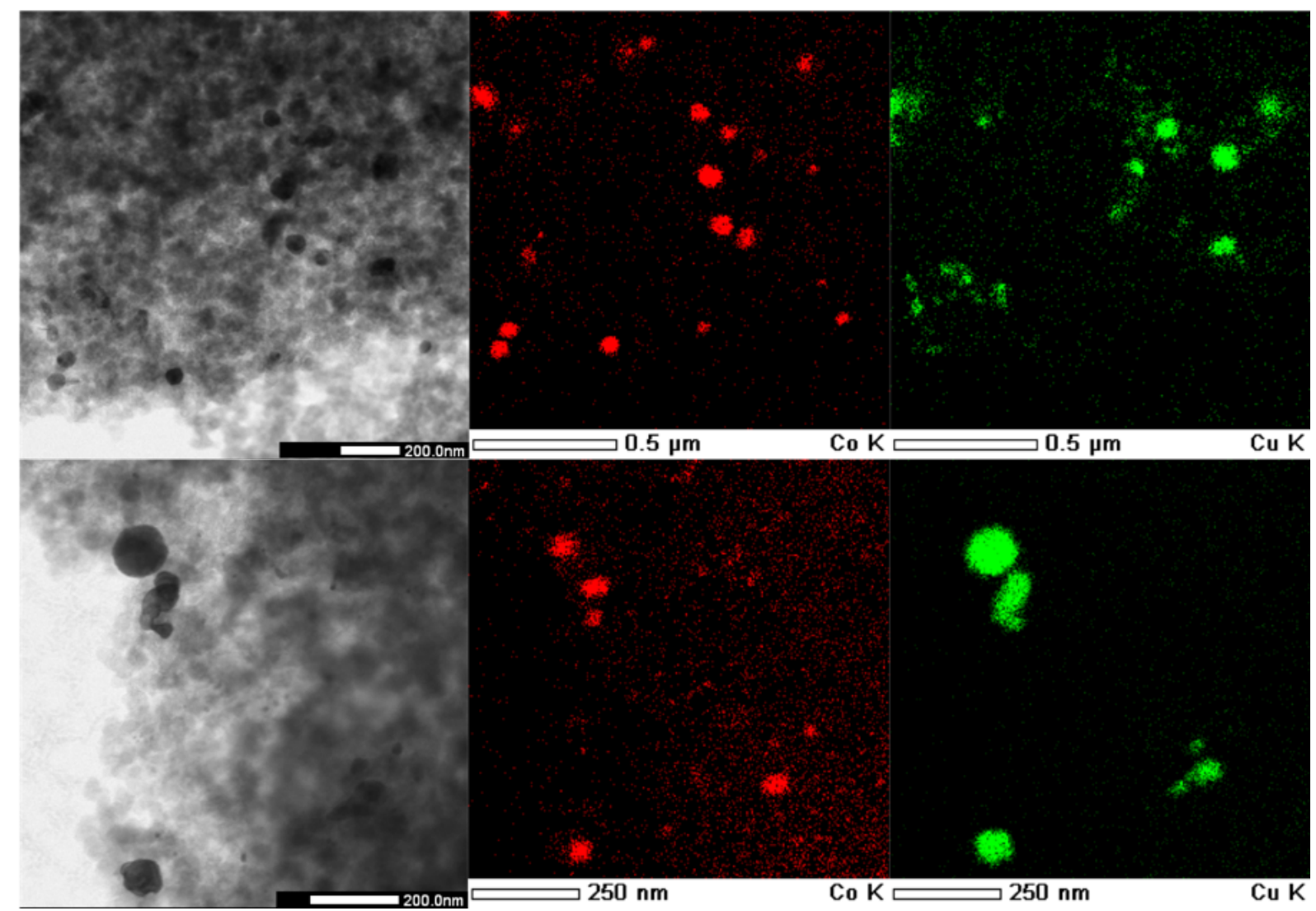

Figure S5. STEM images and EDS mapping of $\mathrm{NCI}-\mathrm{Co} / \mathrm{Cu} 4$

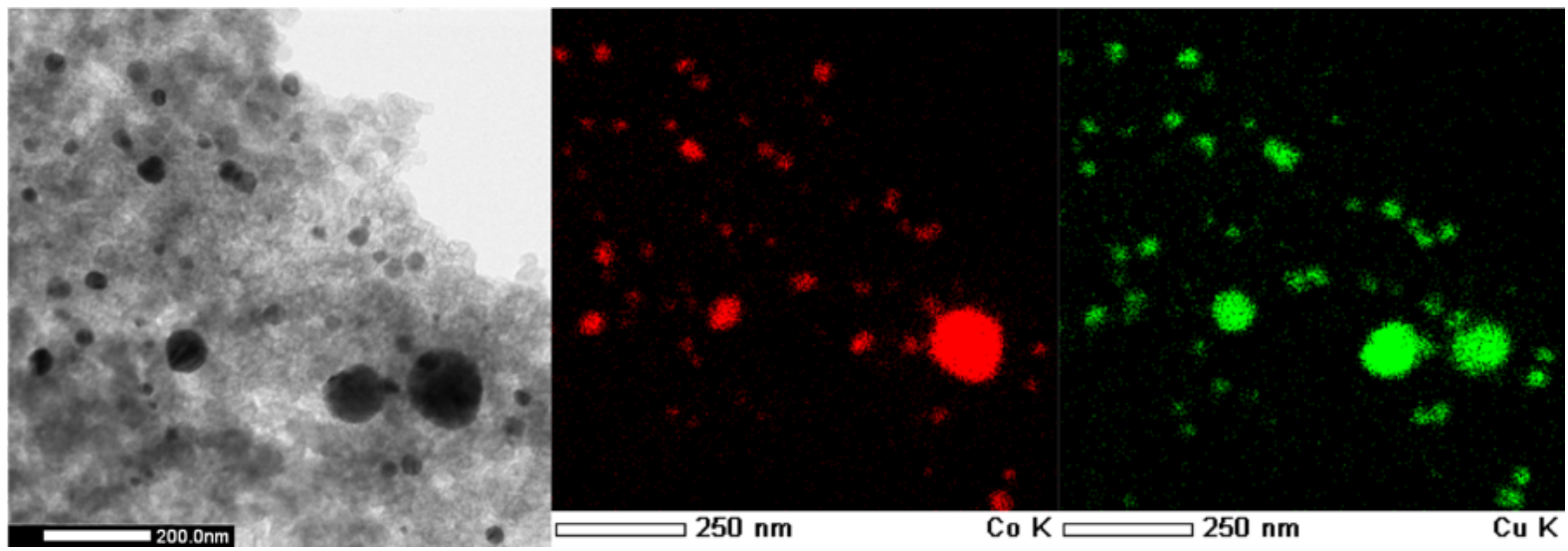

Figure S6. STEM images and EDS mapping of NCI-Co-Cu 13

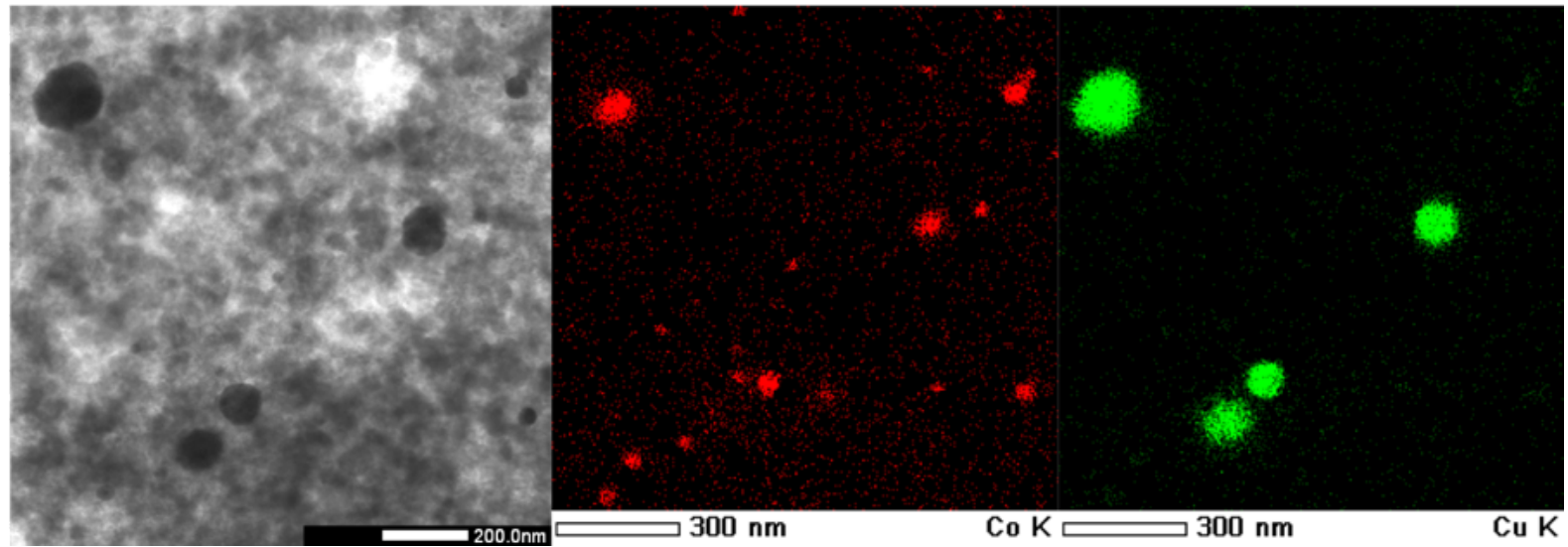

Figure S7. STEM images and EDS mapping of NCI-Cu-Co 14 


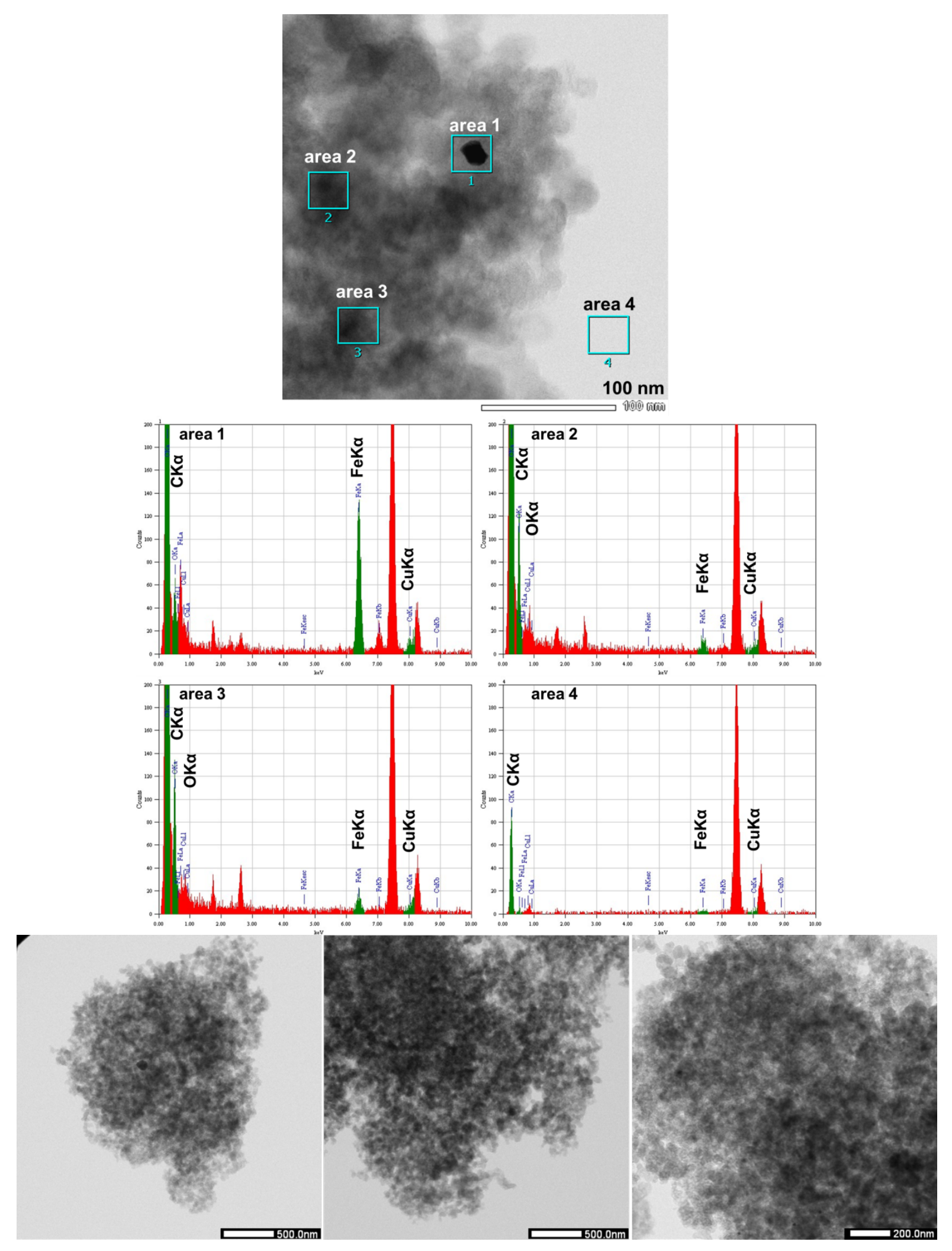

Figure S8. STEM images and area analysis of NCI-Fe/Cu 9-H

EDS analysis of four areas shown as a blue squares in the top picture was conducted. The peaks derived from $\mathrm{CK} \alpha, \mathrm{OK} \alpha, \mathrm{FeK} \alpha, \mathrm{CuK} \alpha$ were highlighted as green peaks. The strongest red peak was derived from the grid (background). 


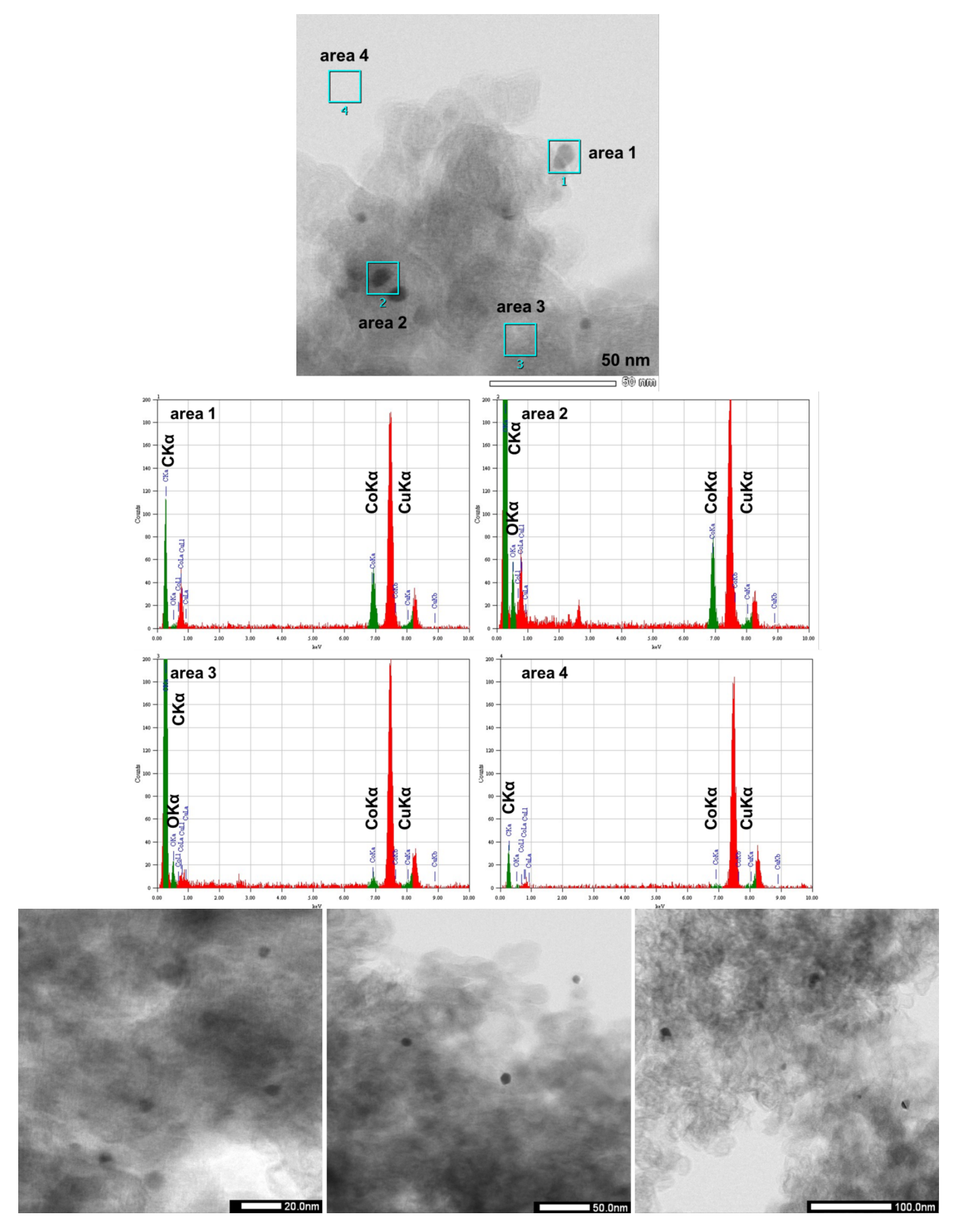

Figure S9. STEM images and EDS analysis of NCI-Co/Cu 4-H

EDS analysis of four areas shown as a blue squares in the top picture was conducted. The peaks derived from $\mathrm{CK} \alpha, \mathrm{OK} \alpha, \mathrm{FeK} \alpha, \mathrm{CuK} \alpha$ were highlighted as green peaks. The strongest red peak was derived from the grid (background). 


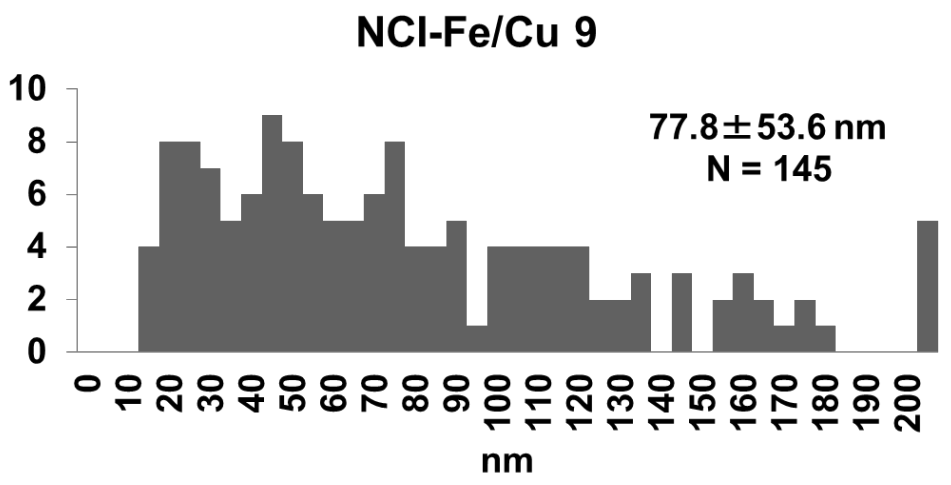

Figure S10. Particle size distribution of NCI-Fe/Cu 9

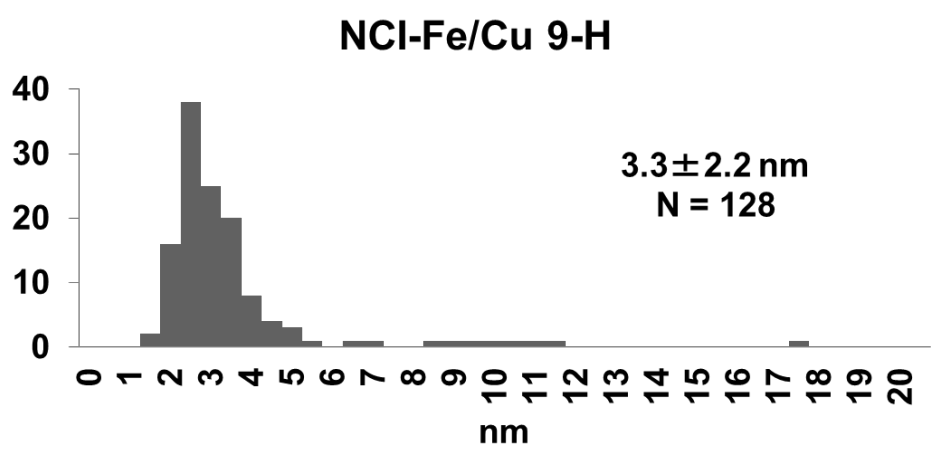

Figure S11. Particle size distribution of NCI-Fe/Cu 9-H

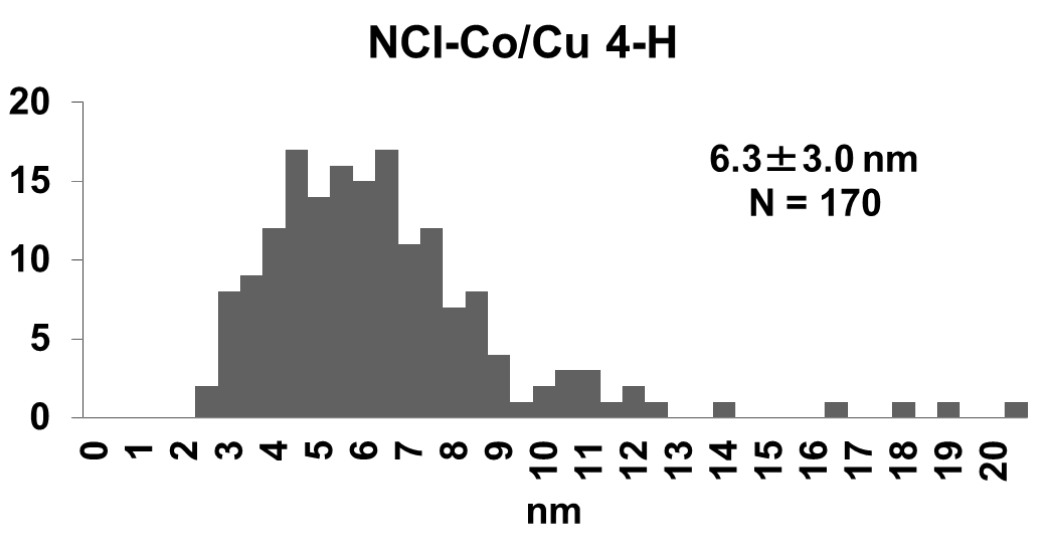

Figure S12. Particle size distribution of NCI-Co/Cu 4-H 


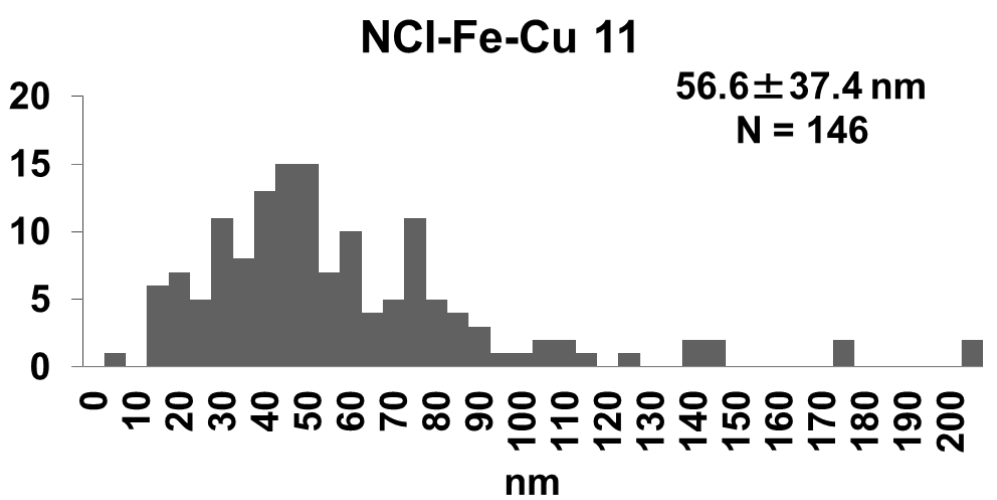

Figure S13. Particle size distribution of NCI-Fe-Cu 11

NCl-Cu-Fe 12

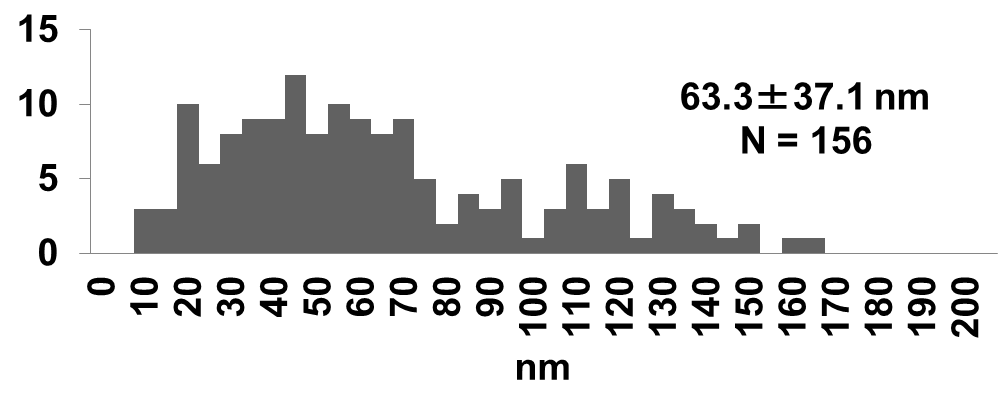

Figure S14. Particle size distribution of NCI-Cu-Fe 12

$\mathrm{NCl}-\mathrm{Co}-\mathrm{Cu} 13$

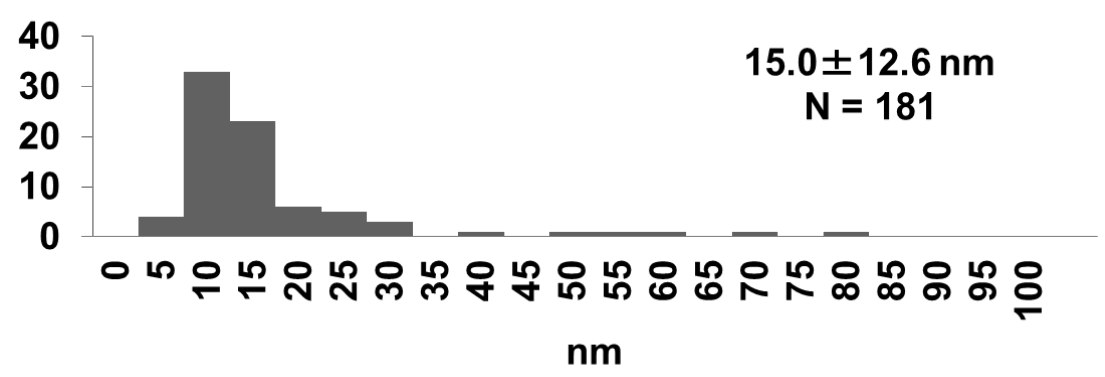

Figure S15. Particle size distribution of NCI-Co-Cu 13

\section{NCl-Cu-Co 14}

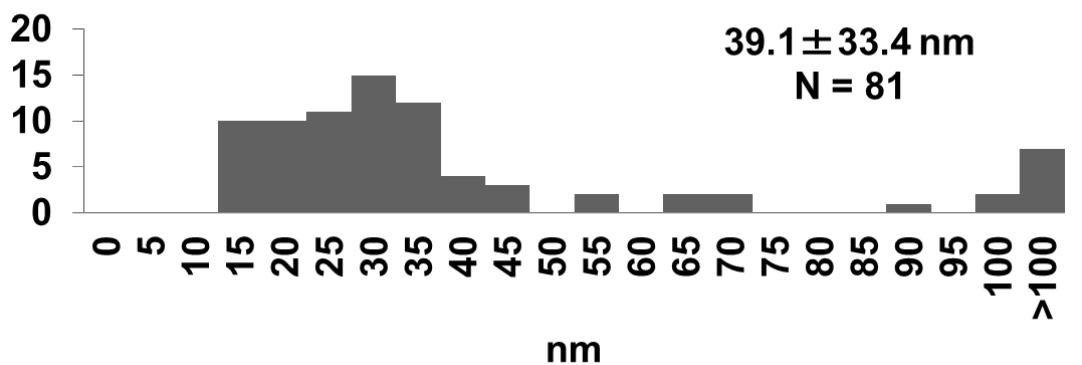

Figure S16. Particle size distribution of NCI-Co-Cu 14 
5. XRD analysis

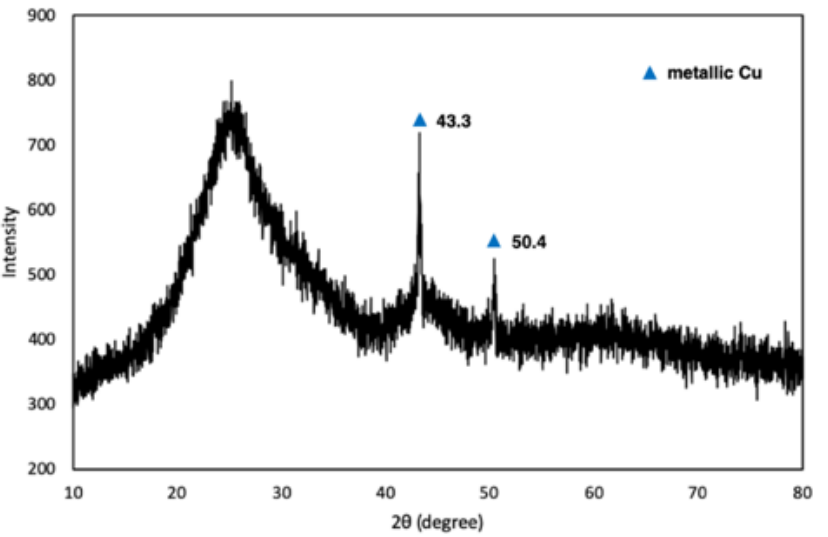

Figure S17. XRD analysis of NCI-Fe/Cu 9

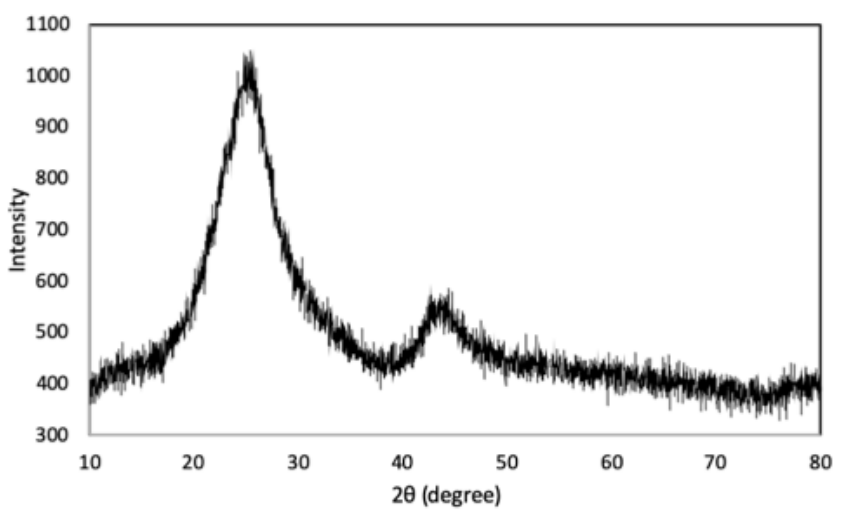

Figure S18. XRD analysis of NCI-Fe/Cu 9-H 


\section{XPS analysis}
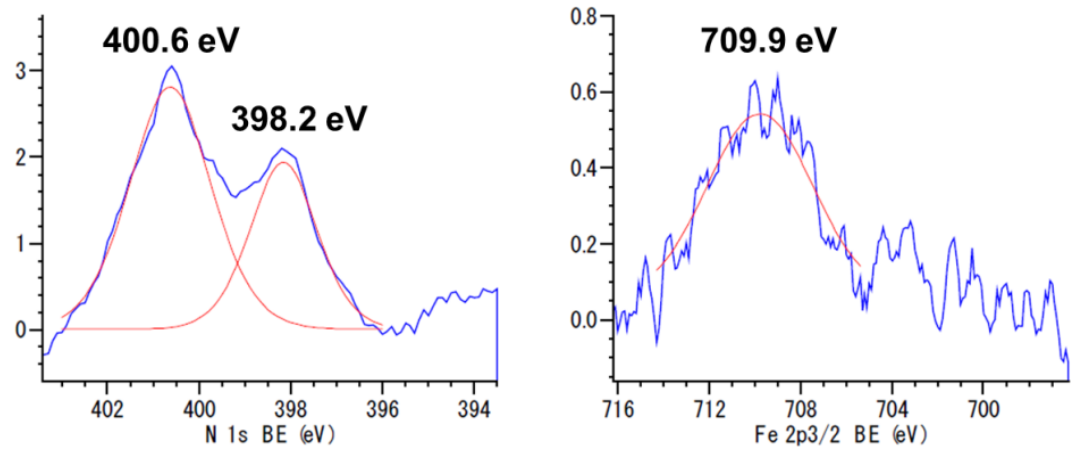

Figure S19. N1s and Fe2p3/2 XPS spectra of NCI-Fe 3
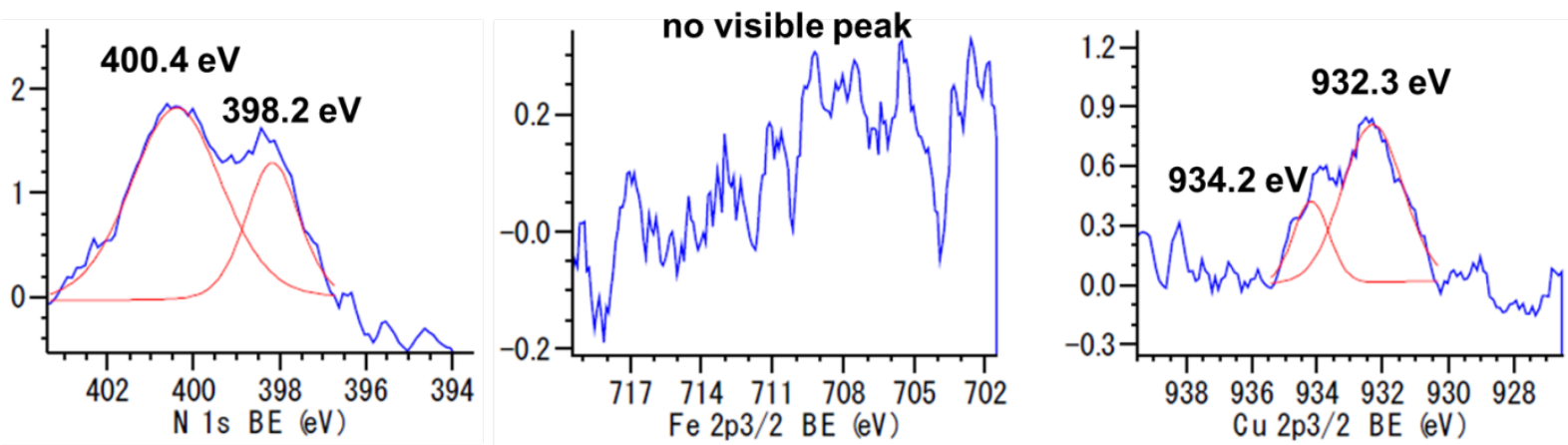

Figure S20. $\mathrm{N} 1 \mathrm{~s}, \mathrm{Fe} 2 \mathrm{p} 3 / 2$ and $\mathrm{Cu} 2 \mathrm{p} 3 / 2$ XPS spectra of NCI-Fe/Cu 9
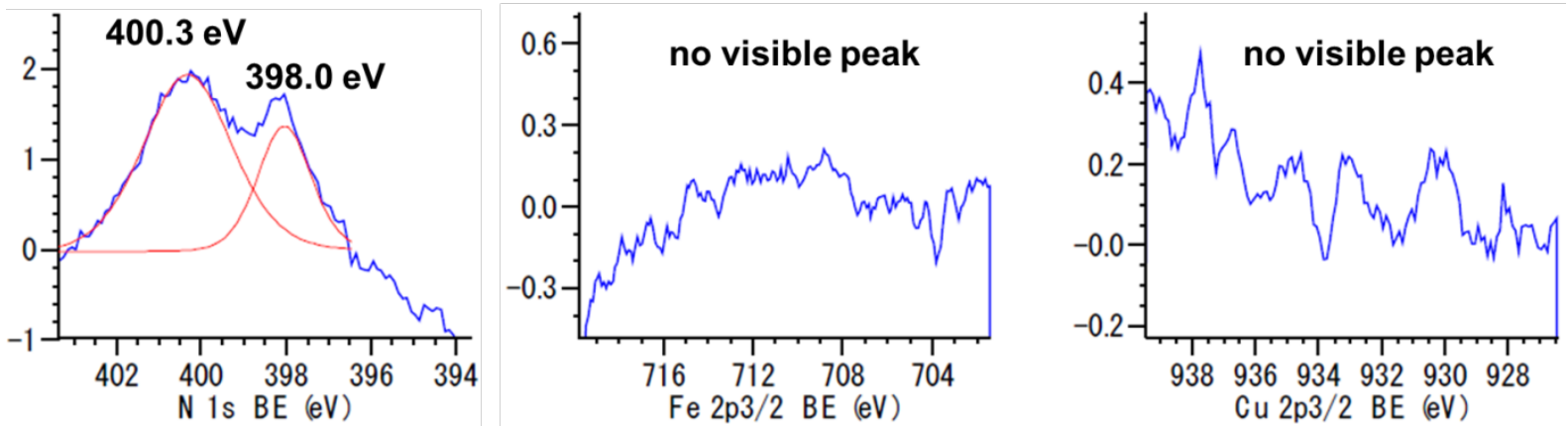

Figure S21. N1s, Fe2p3/2 and $\mathrm{Cu} 2 \mathrm{p} 3 / 2$ XPS spectra of NCI-Fe/Cu 9-H
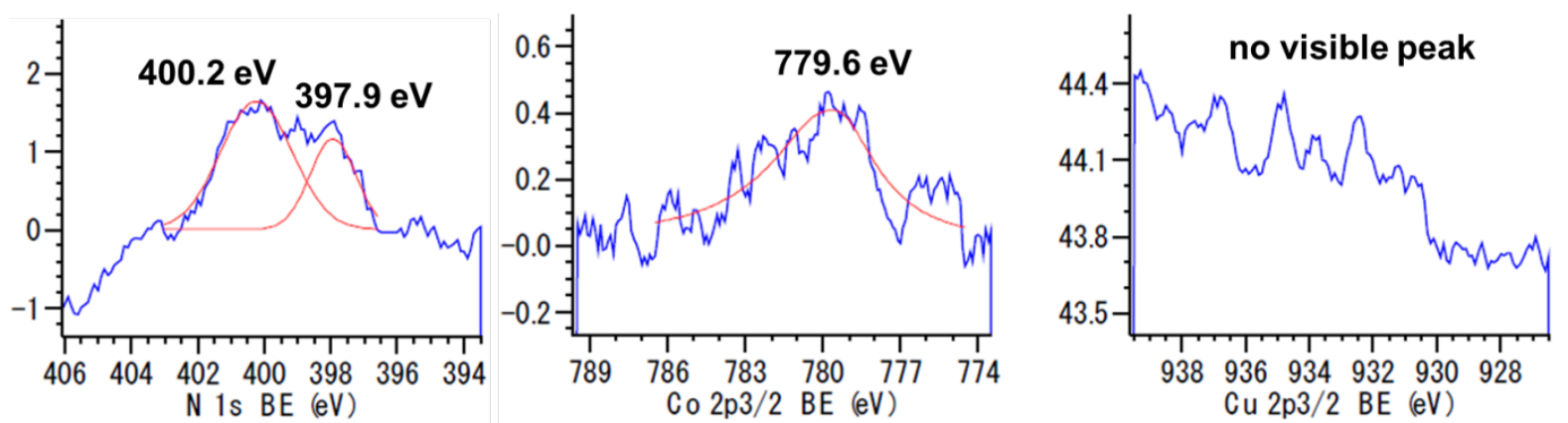

Figure S22. N1s, Co2p3/2 and $\mathrm{Cu} 2 \mathrm{p} 3 / 2$ XPS spectra of NCI-Co/Cu 4-H 
7. NMR charts

4-Chlorobenzonitrile (2b, $\left.\mathrm{CDCl}_{3}, 600 \mathrm{MHz}\right)$

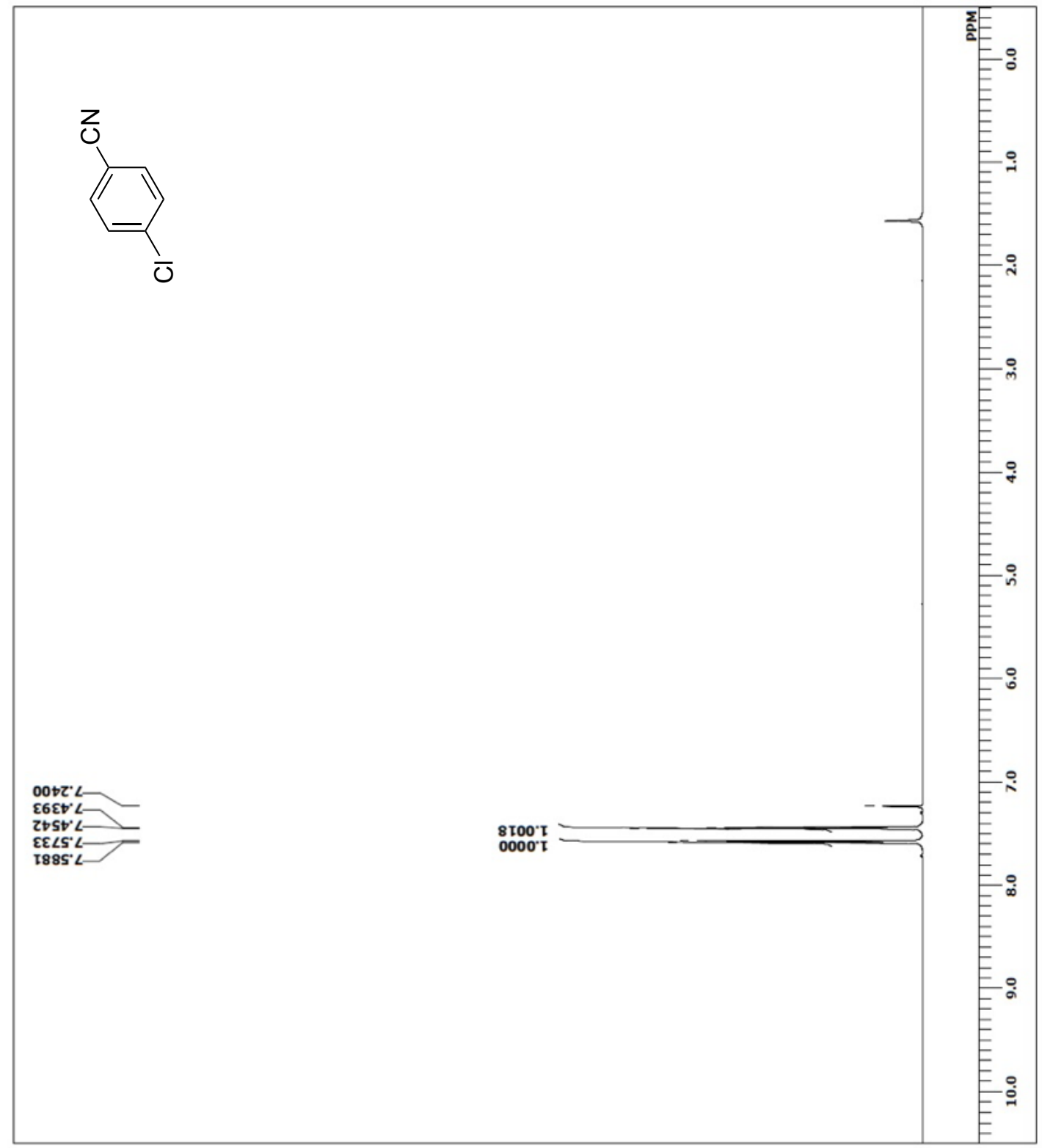


4-Chlorobenzonitrile (2b, $\left.\mathrm{CDCl}_{3}, 150 \mathrm{MHz}\right)$

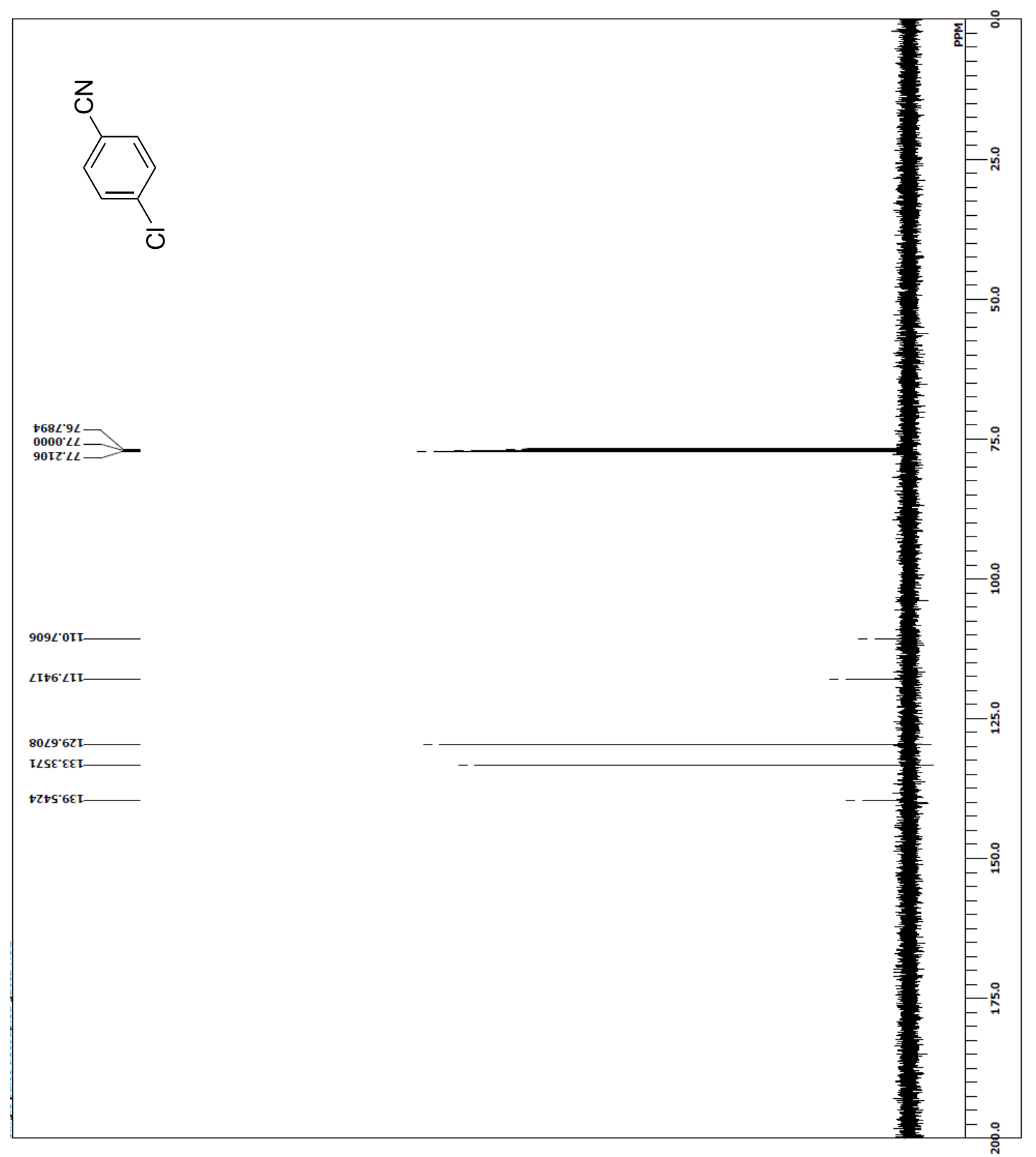


4-Bromobenzonitrile (2c, $\left.\mathrm{CDCl}_{3}, 600 \mathrm{MHz}\right)$

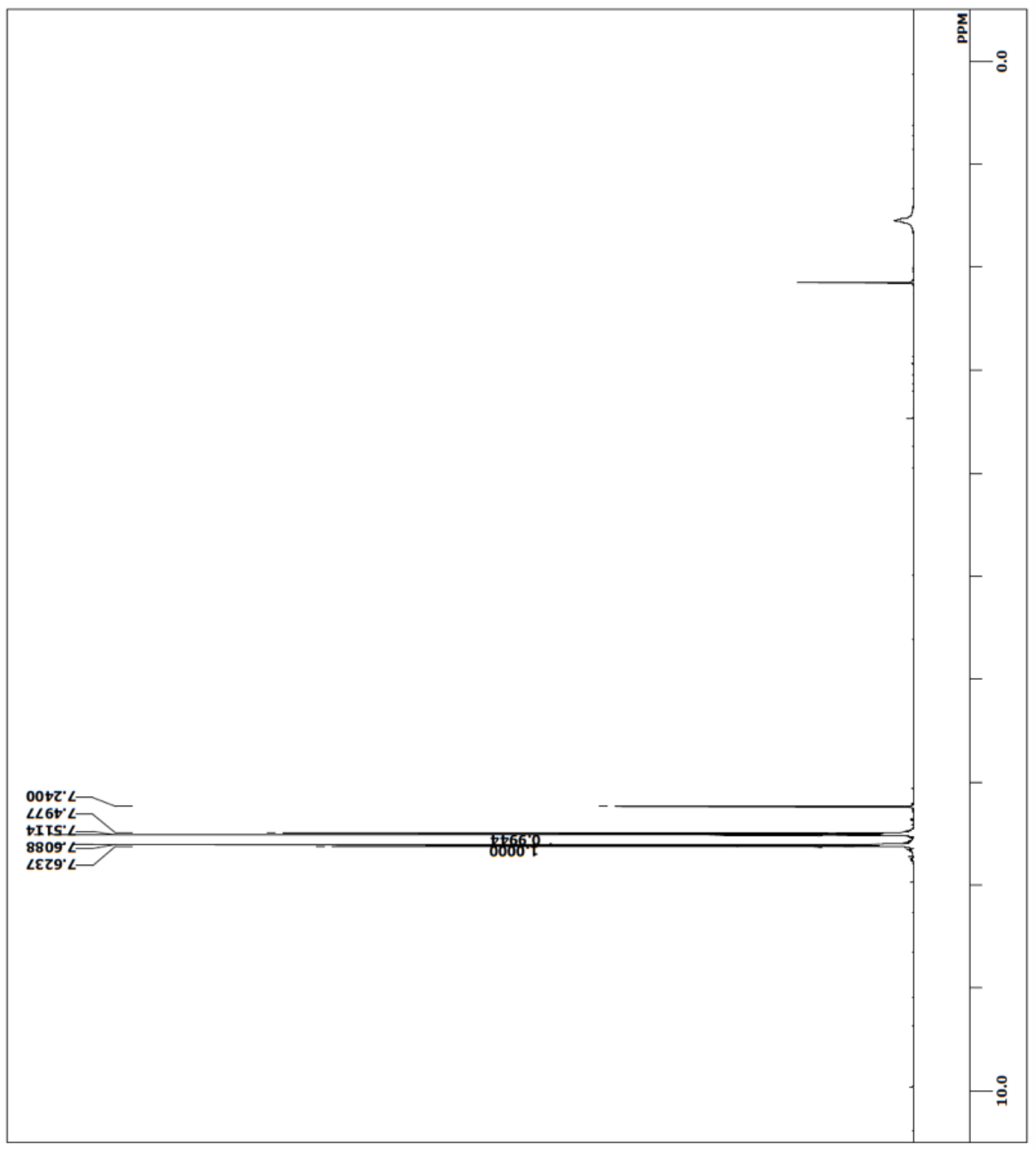


4-Bromobenzonitrile (2c, $\left.\mathrm{CDCl}_{3}, 150 \mathrm{MHz}\right)$

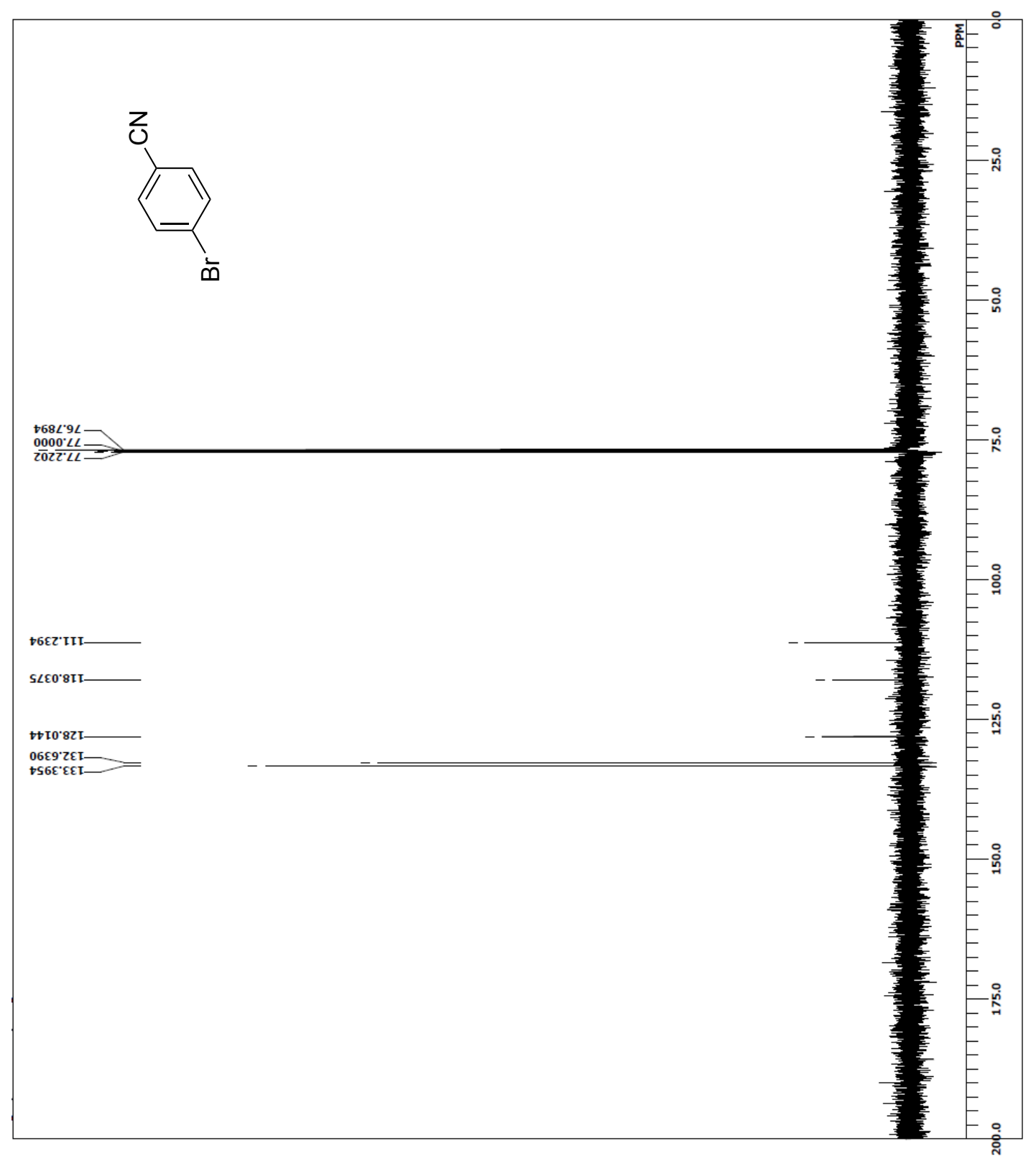


4-Nitrobenzonitrile (2d, $\left.\mathrm{CDCl}_{3}, 600 \mathrm{MHz}\right)$

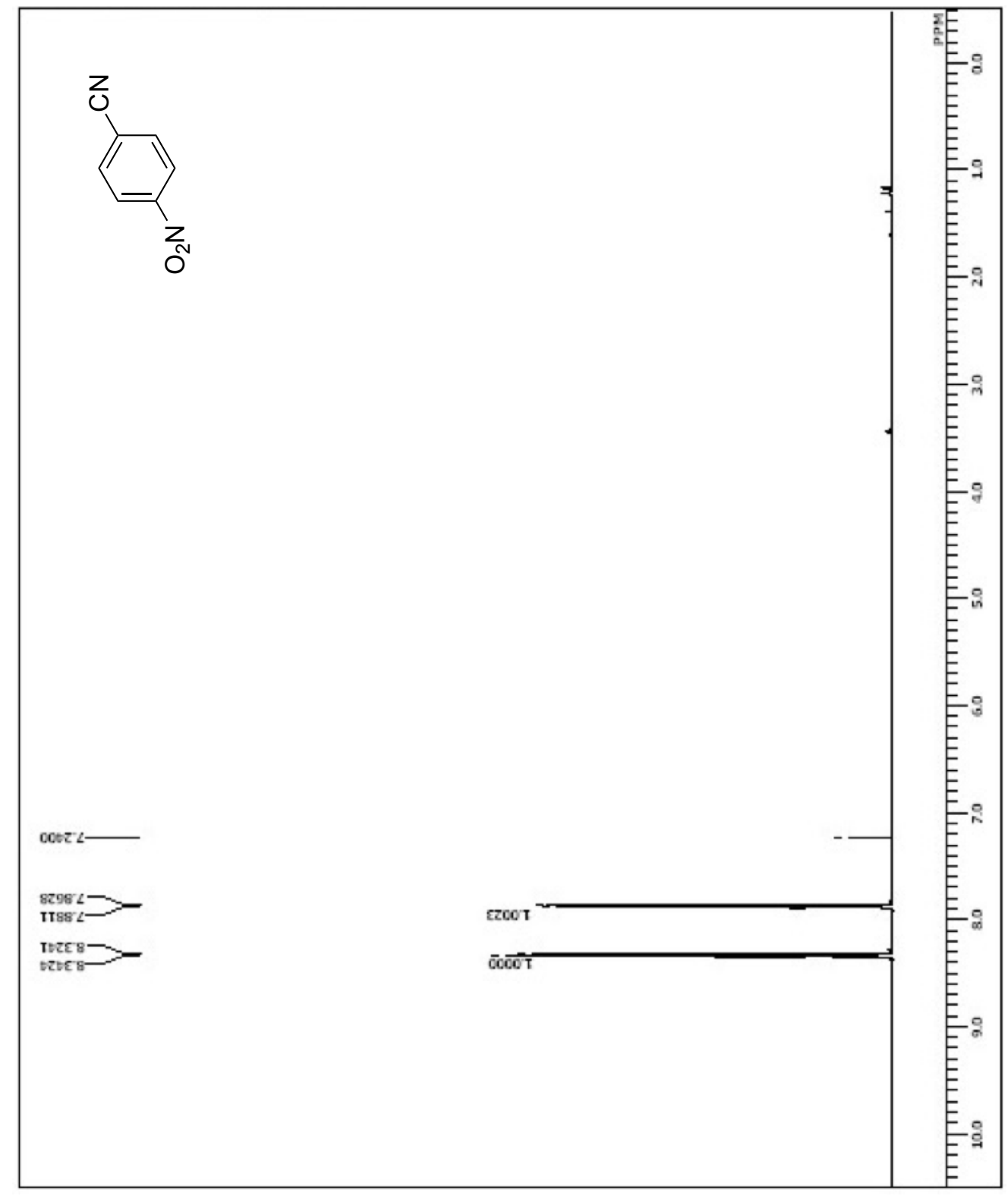


4-Nitrobenzonitrile (2d, $\left.\mathrm{CDCl}_{3}, 150 \mathrm{MHz}\right)$

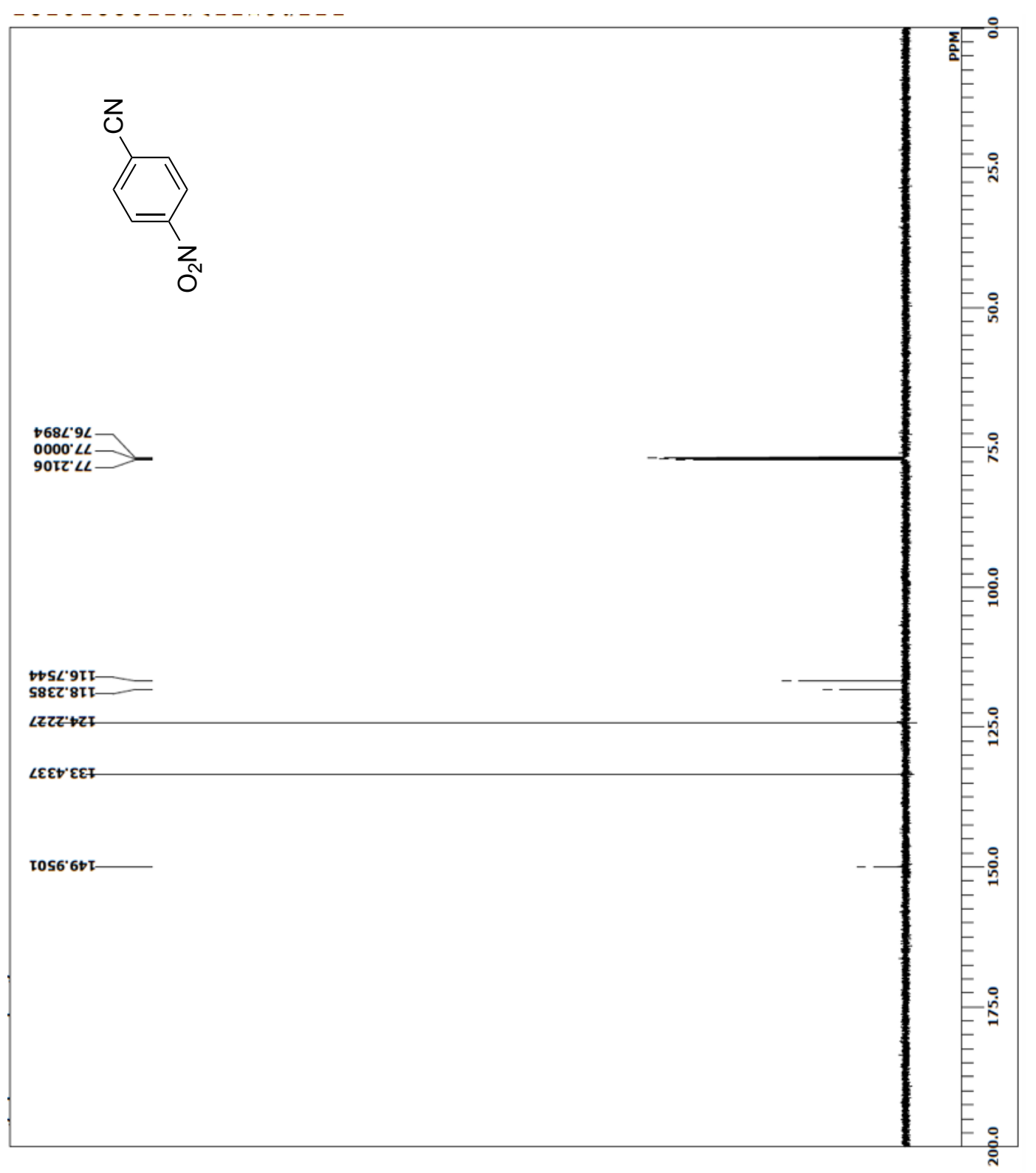


4-Methylbenzonitrile (2e, $\left.\mathrm{CDCl}_{3}, 600 \mathrm{MHz}\right)$

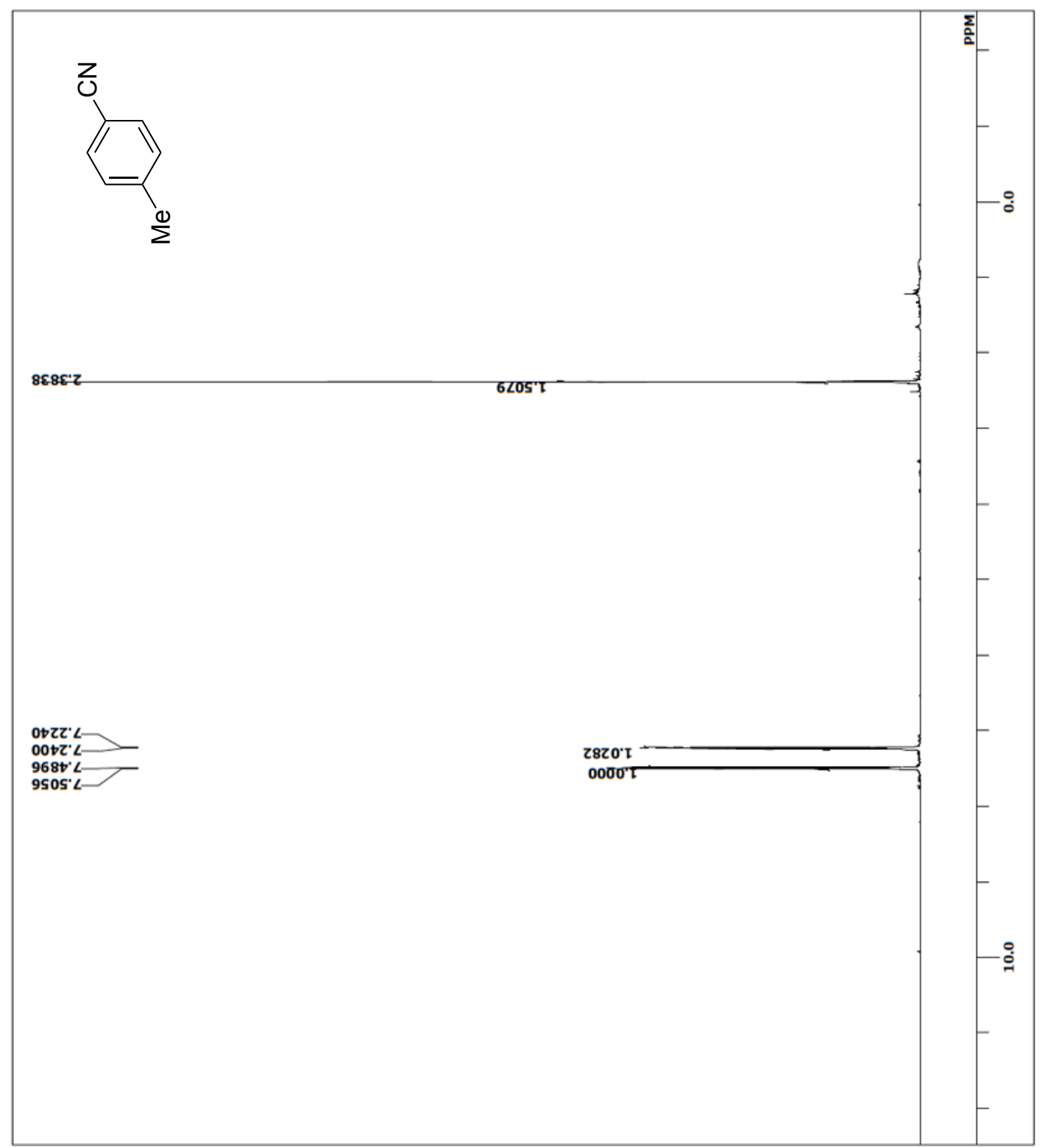


4-Methylbenzonitrile (2e, $\left.\mathrm{CDCl}_{3}, 150 \mathrm{MHz}\right)$

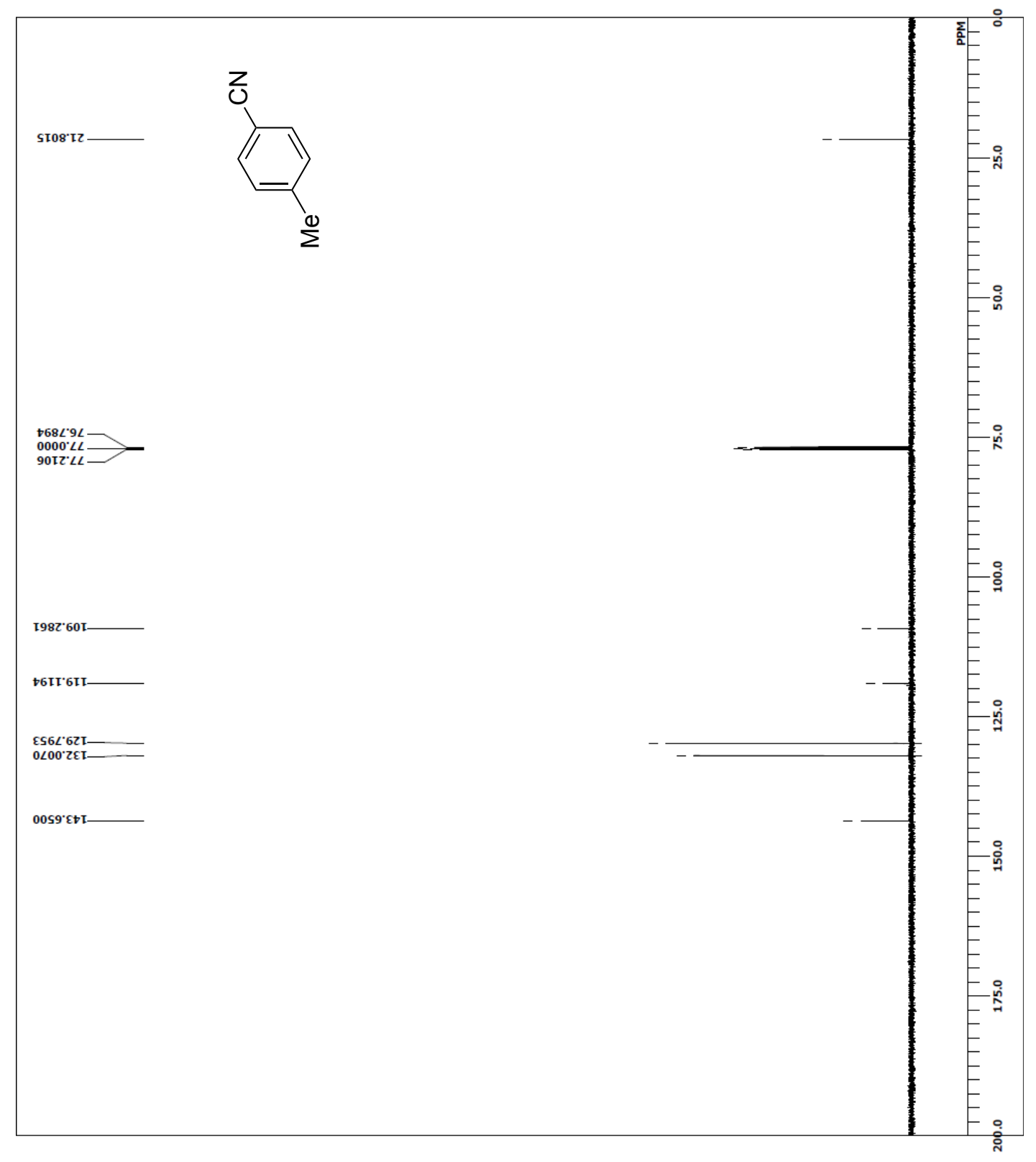

S23 
4-Methoxylbenzonitrile (2f, $\mathrm{CDCl}_{3}, 600 \mathrm{MHz}$ )

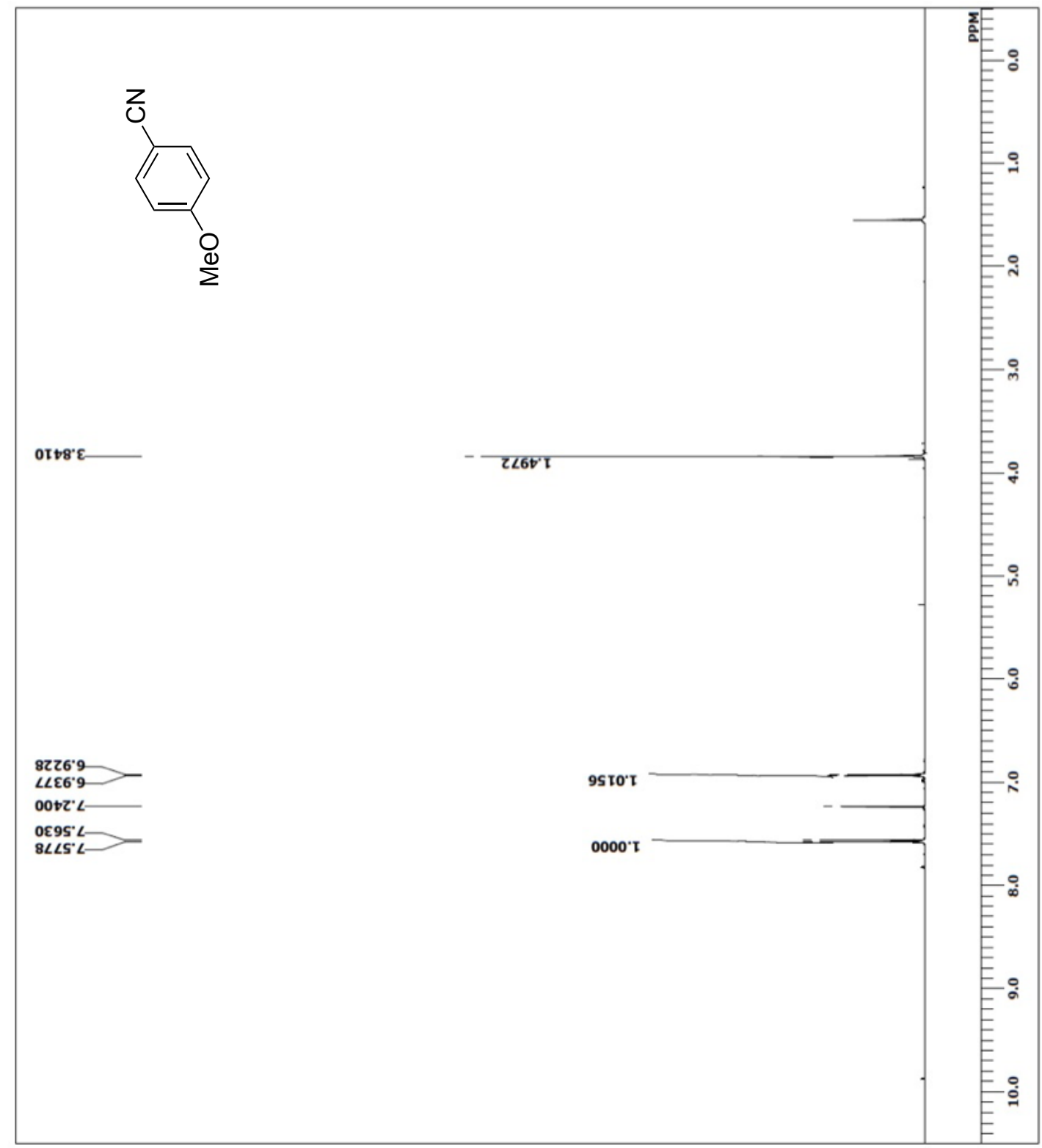


4-Methoxylbenzonitrile (2f, $\mathrm{CDCl}_{3}, 150 \mathrm{MHz}$ )

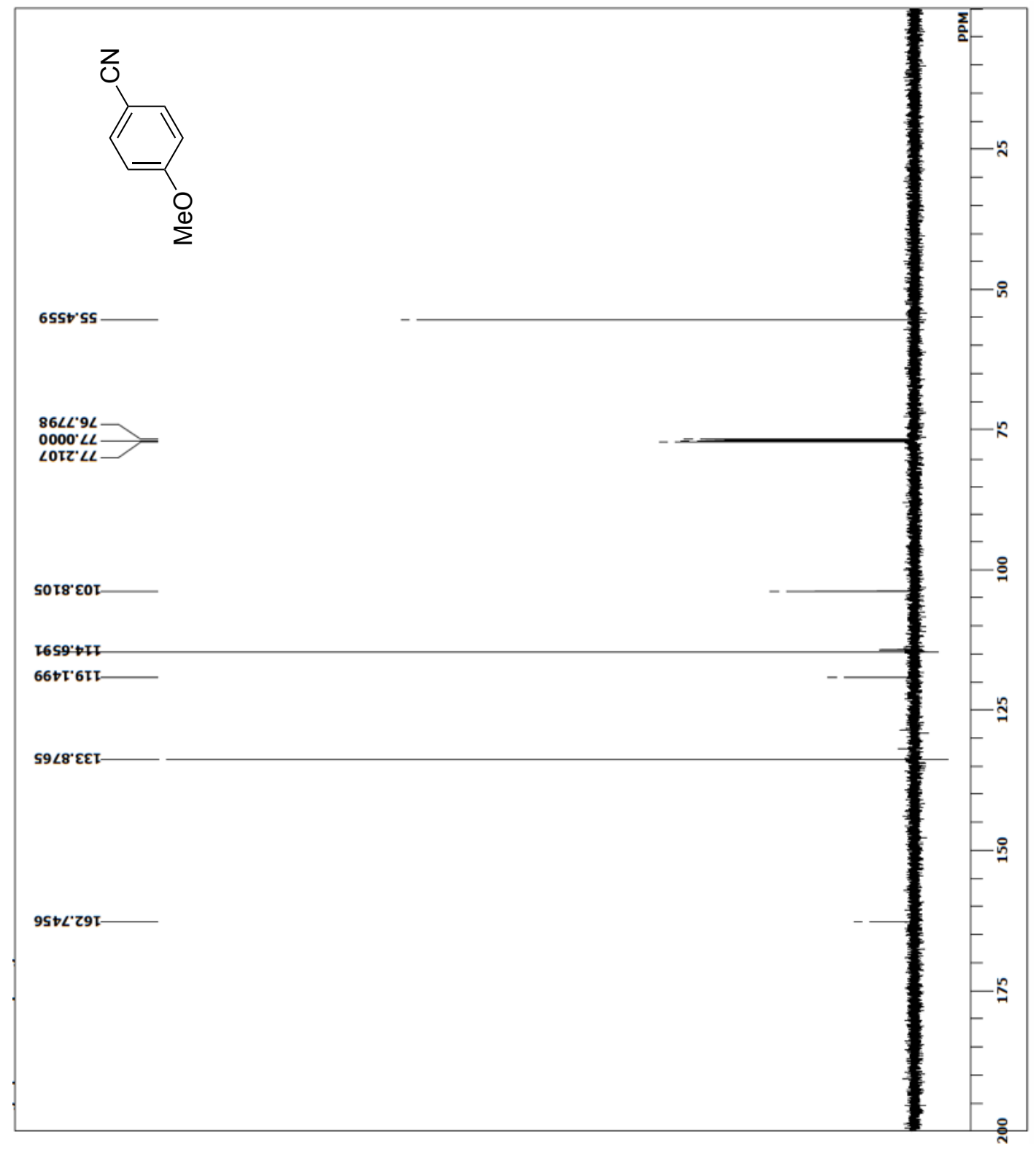


3-Methoxylbenzonitrile (2g, $\left.\mathrm{CDCl}_{3}, 600 \mathrm{MHz}\right)$

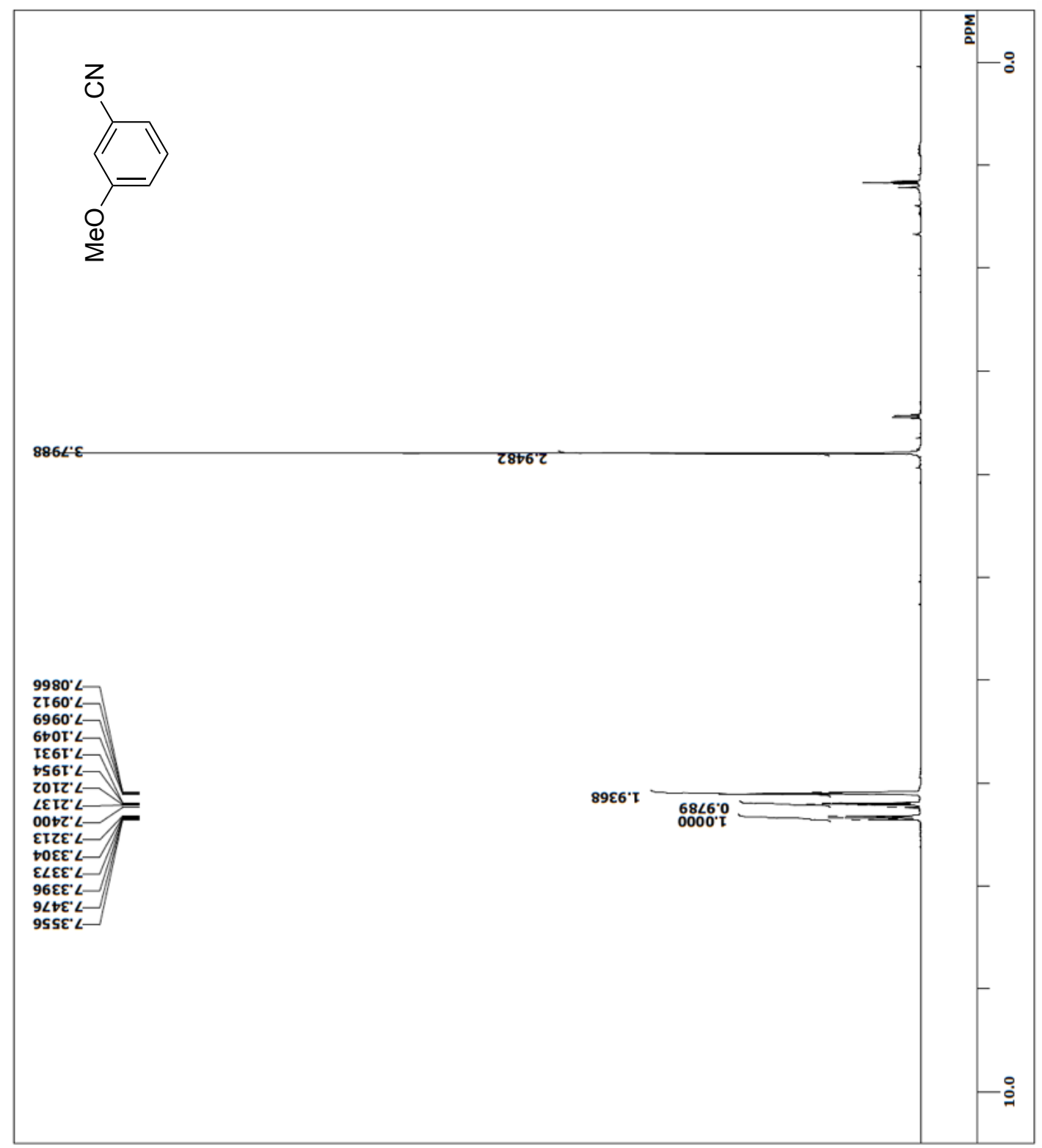


3-Methoxylbenzonitrile (2g, $\left.\mathrm{CDCl}_{3}, 150 \mathrm{MHz}\right)$

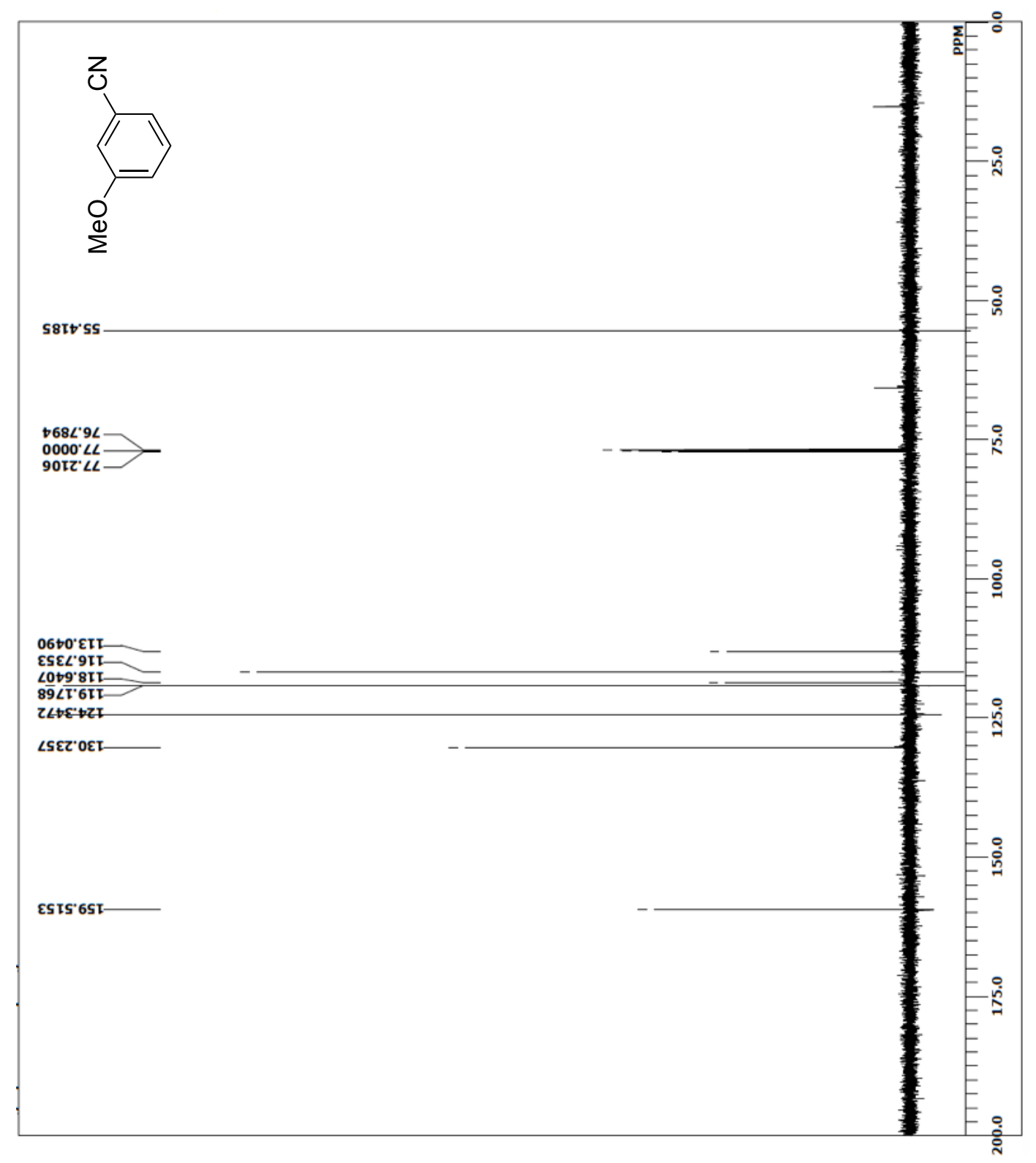


2-Methoxylbenzonitrile (2h, $\left.\mathrm{CDCl}_{3}, 600 \mathrm{MHz}\right)$

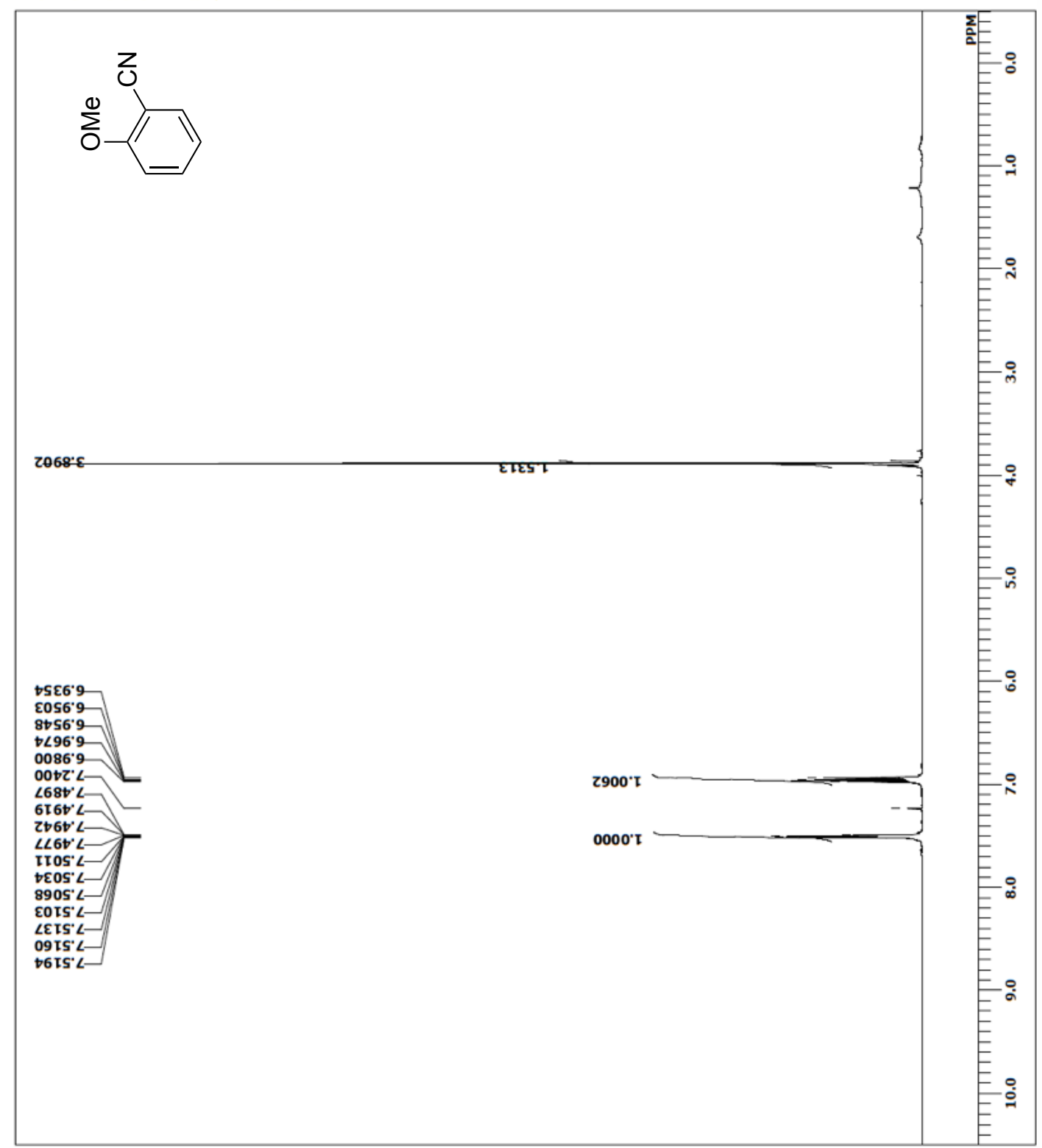


2-Methoxylbenzonitrile (2h, $\left.\mathrm{CDCl}_{3}, 150 \mathrm{MHz}\right)$

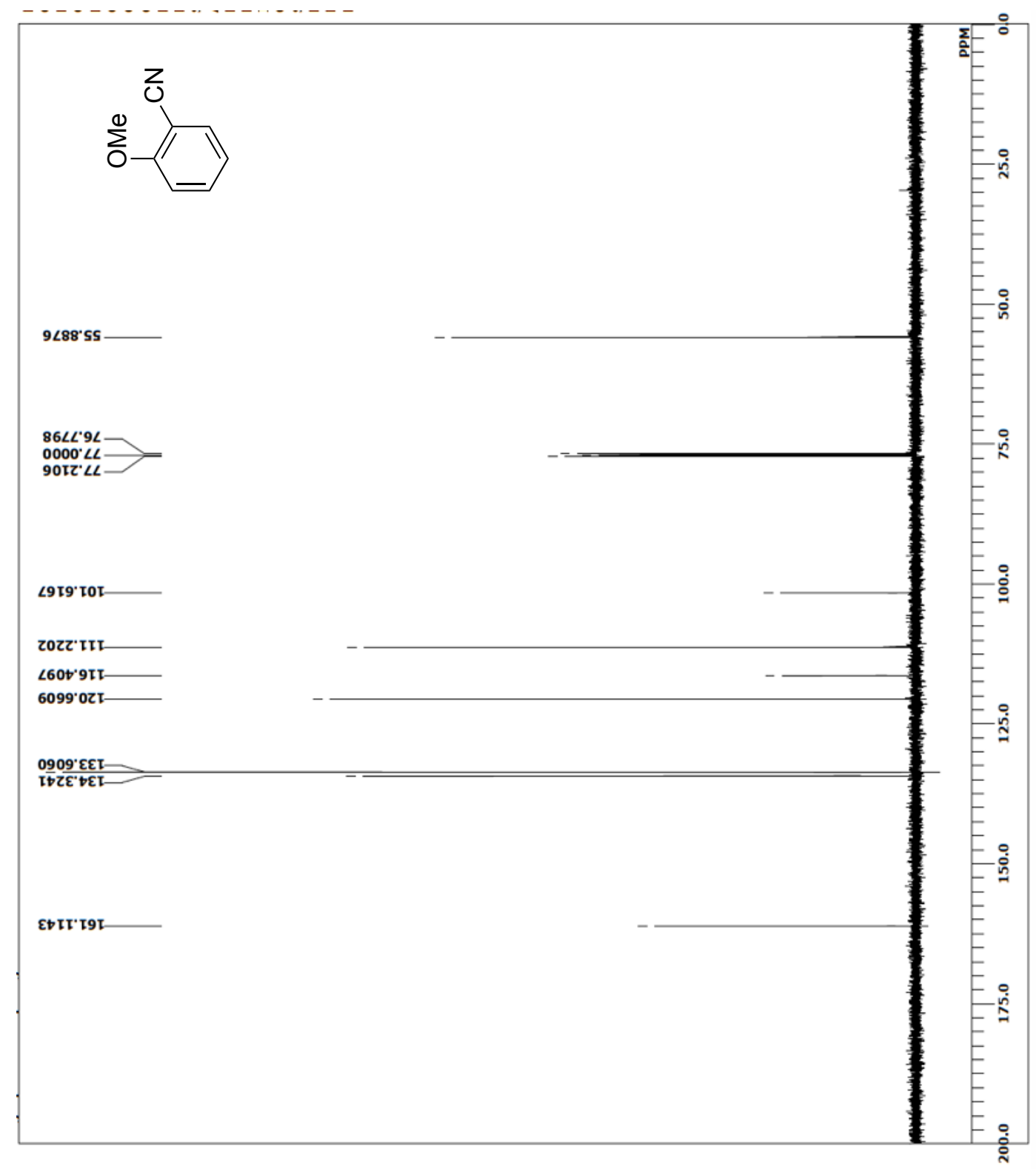


2-Cyanonaphthalene (2i, $\left.\mathrm{CDCl}_{3}, 600 \mathrm{MHz}\right)$

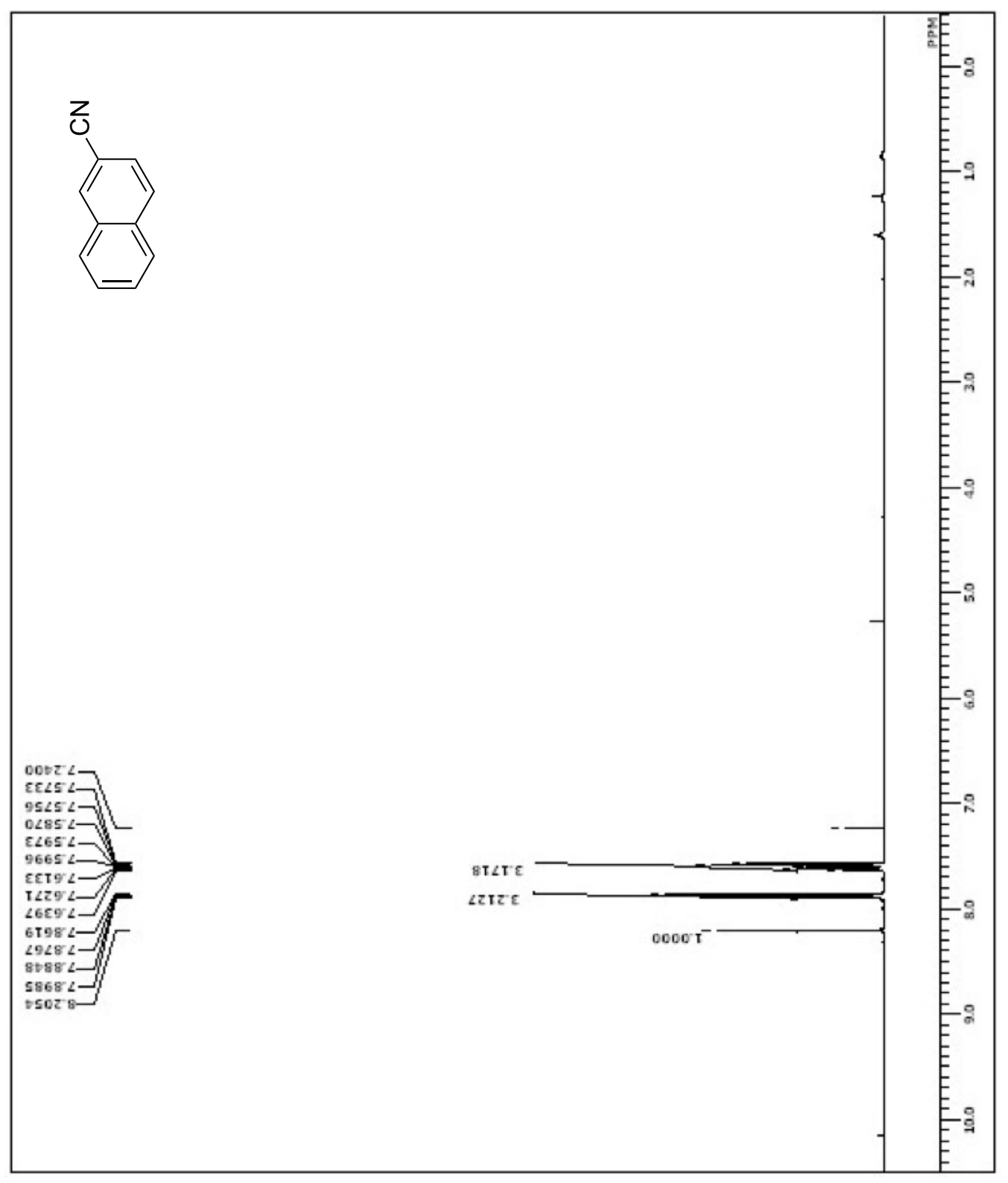


2-Cyanonaphthalene (2i, $\left.\mathrm{CDCl}_{3}, 150 \mathrm{MHz}\right)$

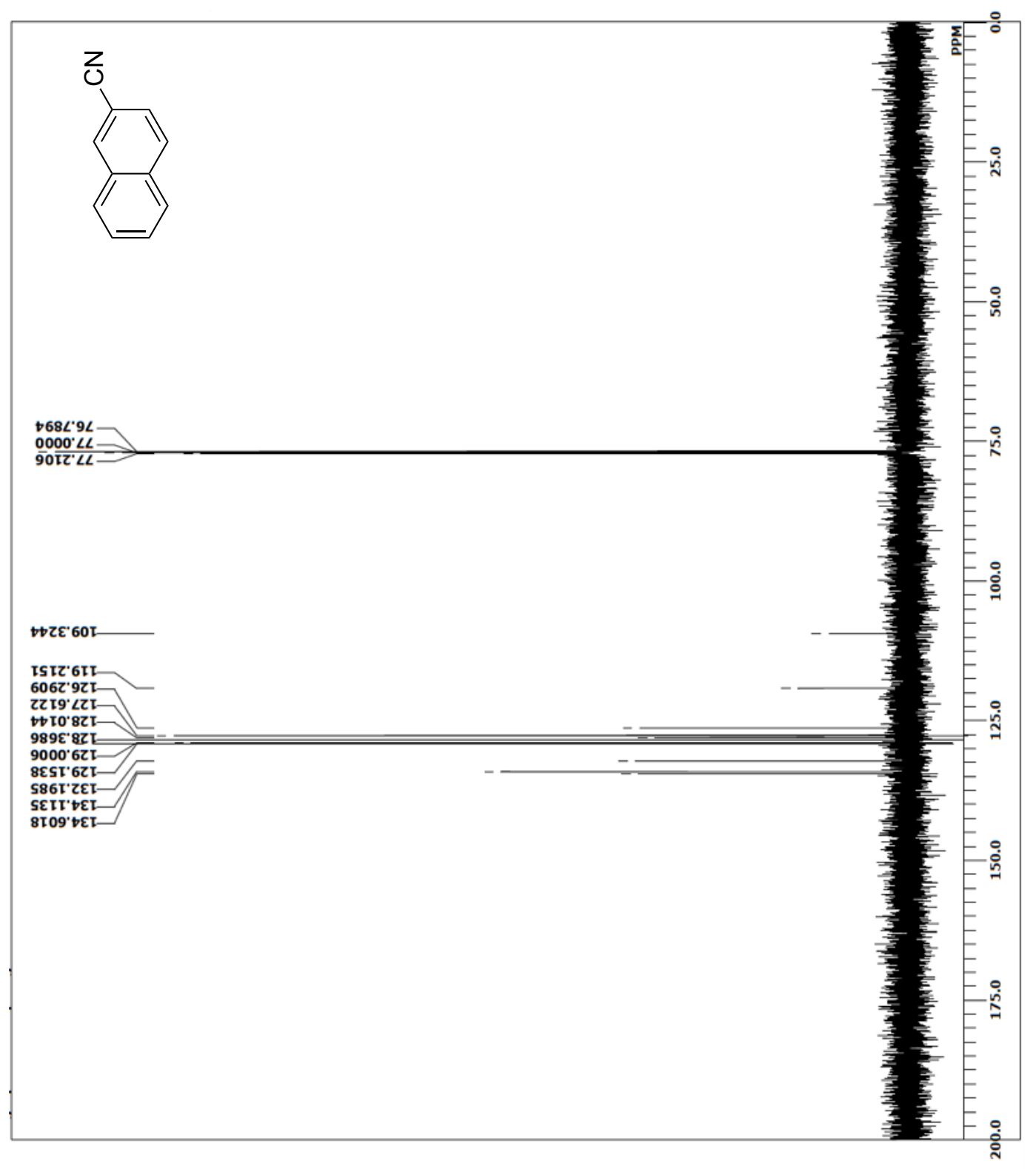


Terephthalonitrile (2j, $\left.\mathrm{CDCl}_{3}, 600 \mathrm{MHz}\right)$

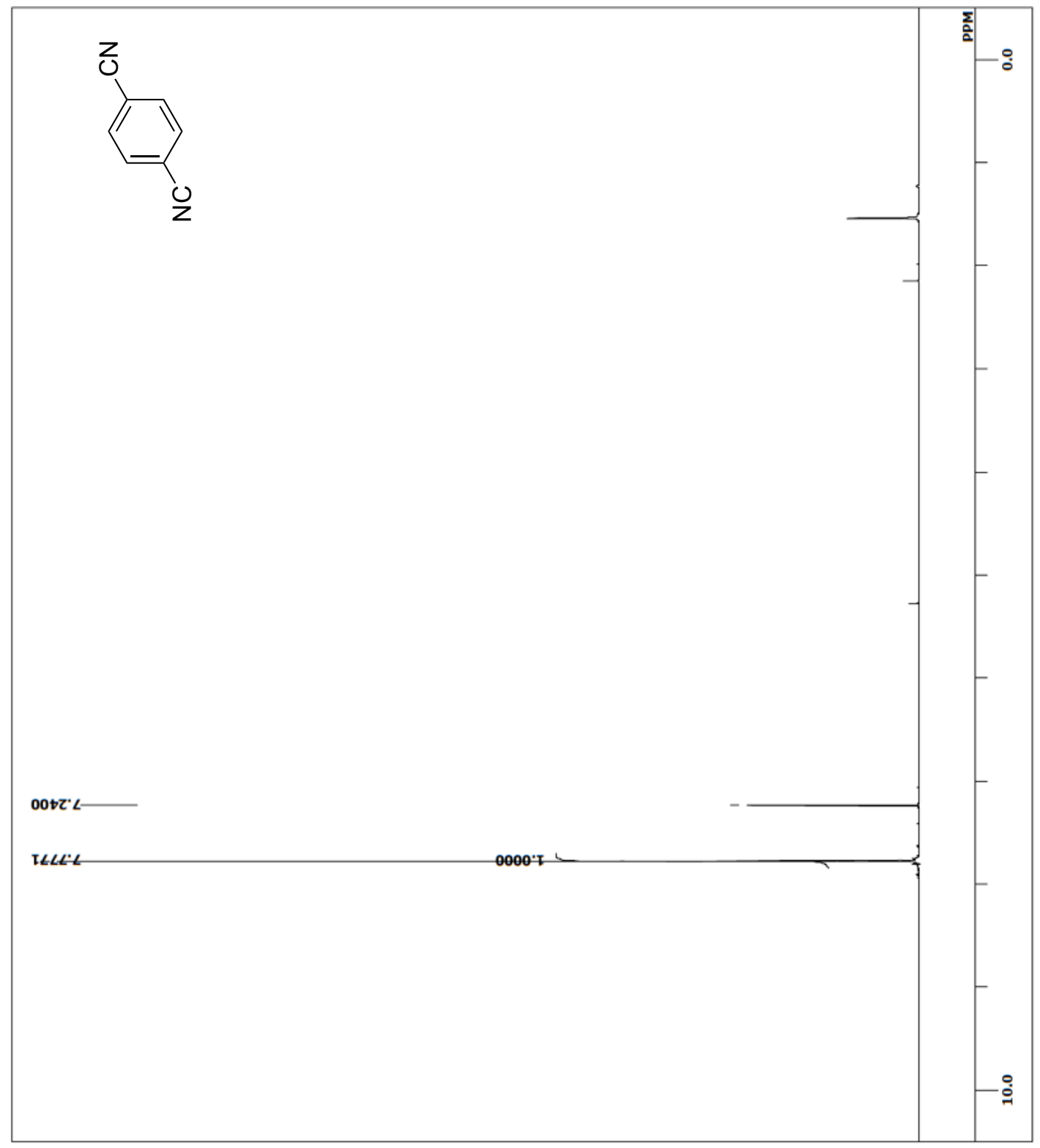


Terephthalonitrile $\left(\mathbf{2} \mathbf{j}, \mathrm{CDCl}_{3}, 150 \mathrm{MHz}\right)$

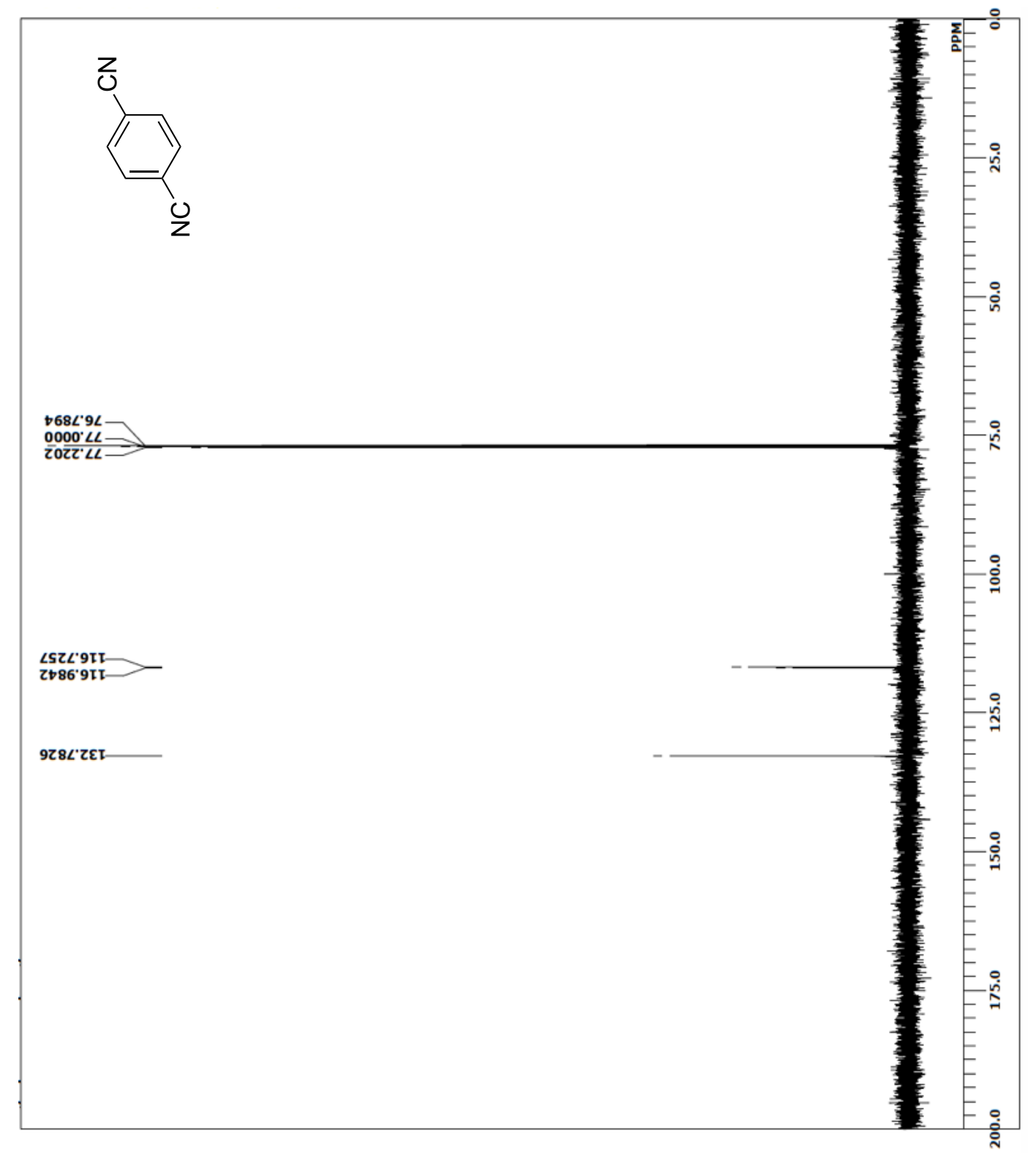


Thiophene-2-carbonitrile (2k, $\left.\mathrm{CDCl}_{3}, 600 \mathrm{MHz}\right)$

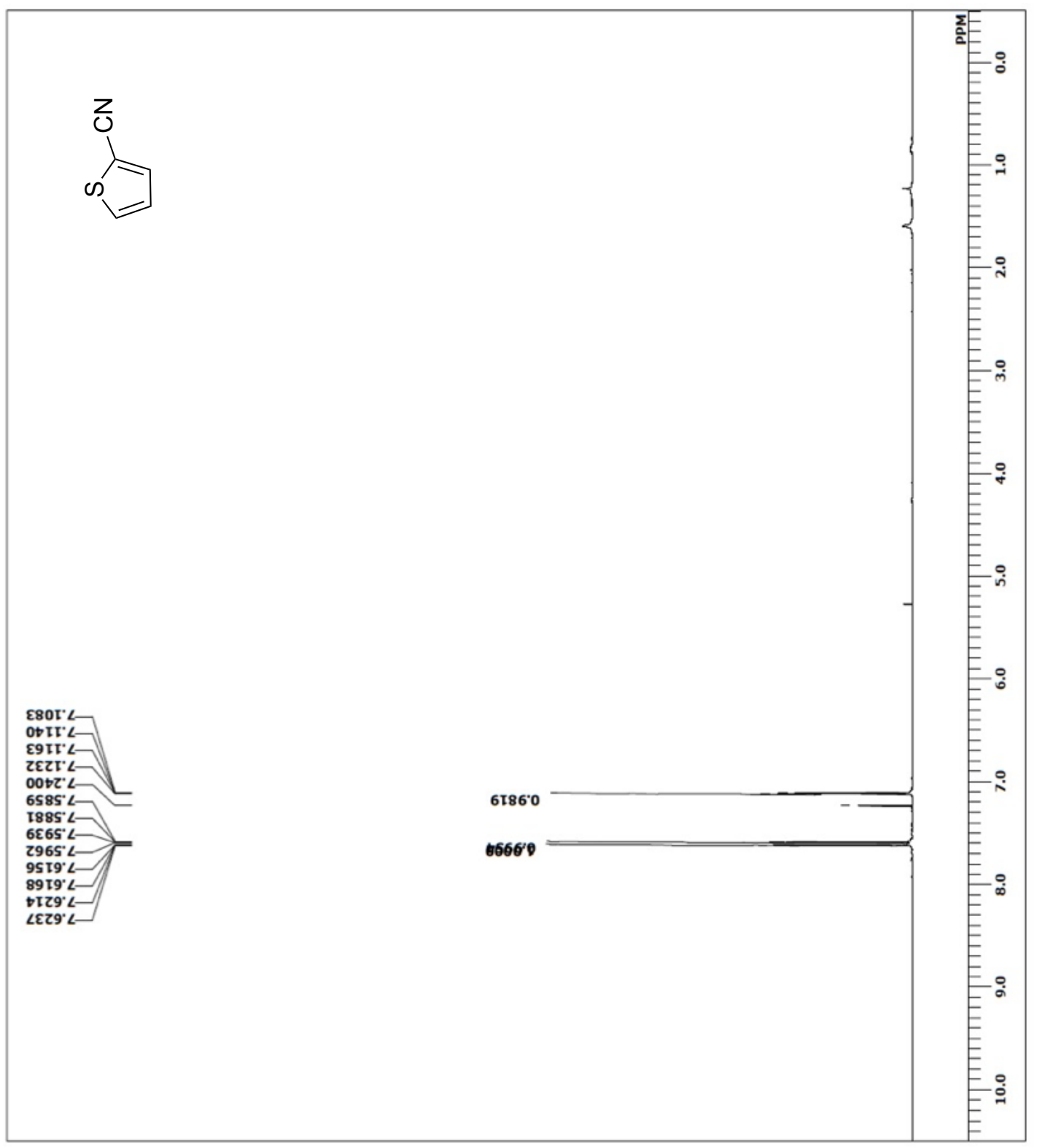


Thiophene-2-carbonitrile (2k, $\left.\mathrm{CDCl}_{3}, 150 \mathrm{MHz}\right)$

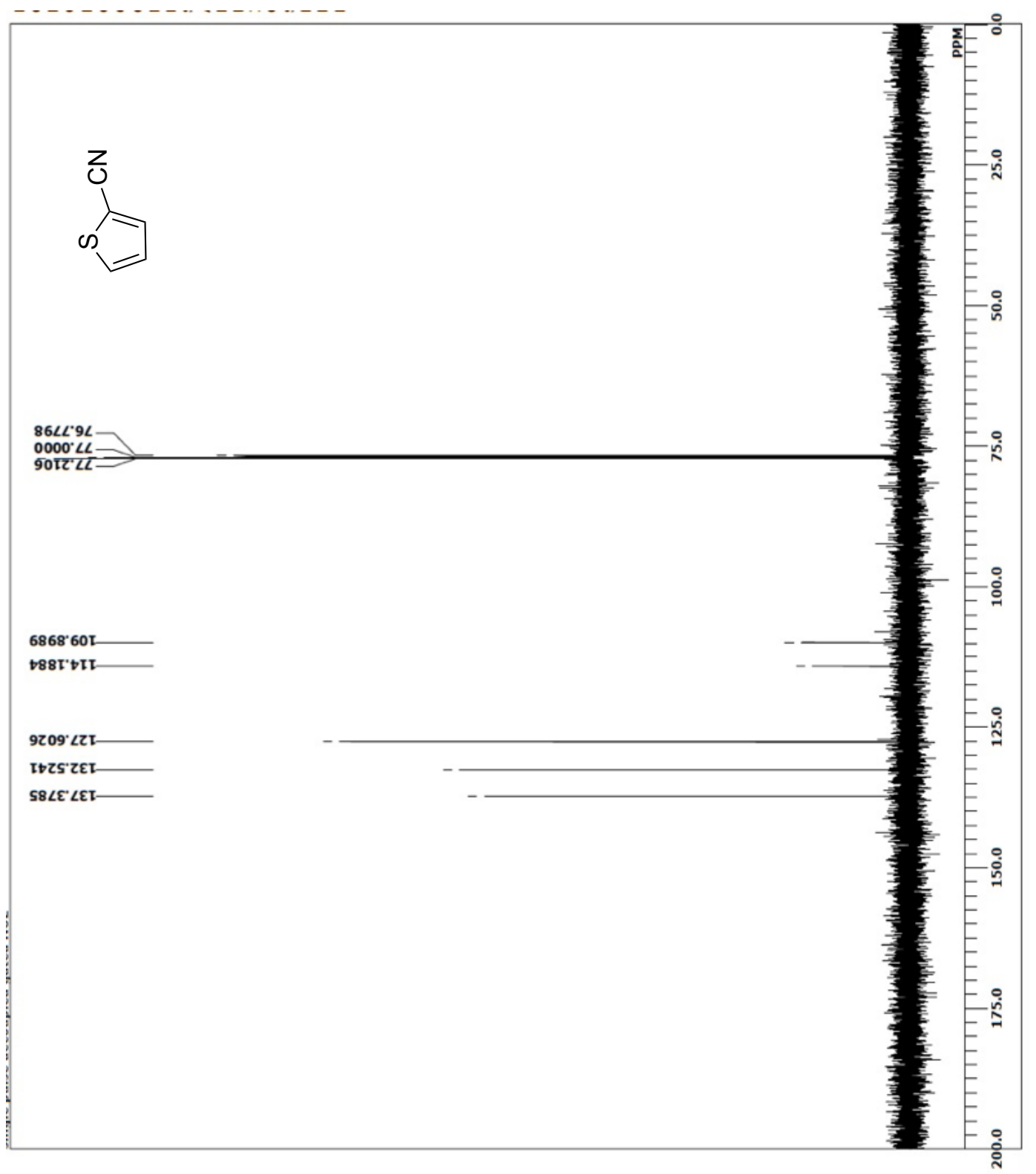


Furan-2-carbonitrile (2l, $\left.\mathrm{CDCl}_{3}, 600 \mathrm{MHz}\right)$

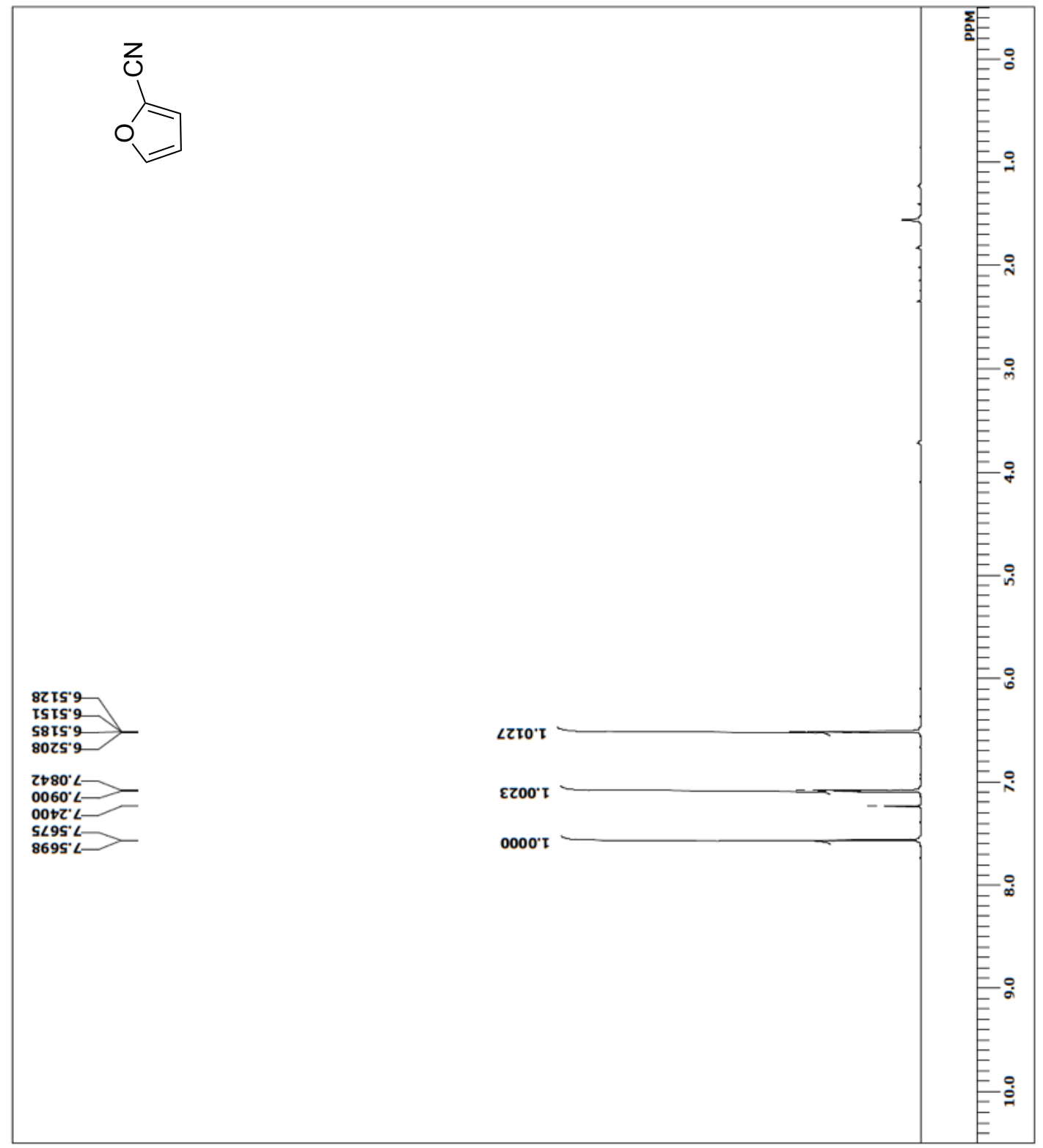


Furan-2-carbonitrile (2l, $\left.\mathrm{CDCl}_{3}, 150 \mathrm{MHz}\right)$

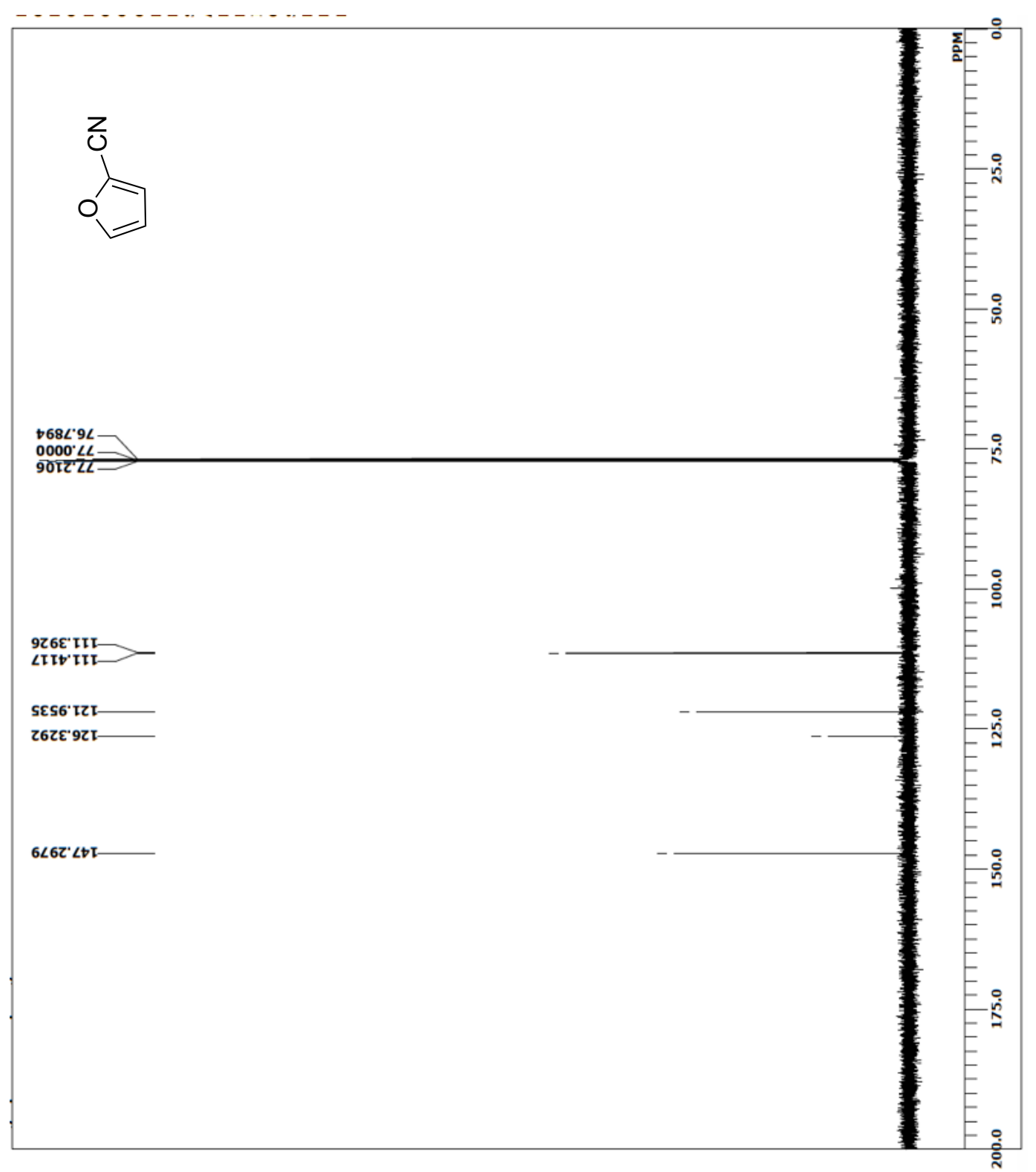


Nicotinonitrile $\left(\mathbf{2 m}, \mathrm{CDCl}_{3}, 600 \mathrm{MHz}\right)$

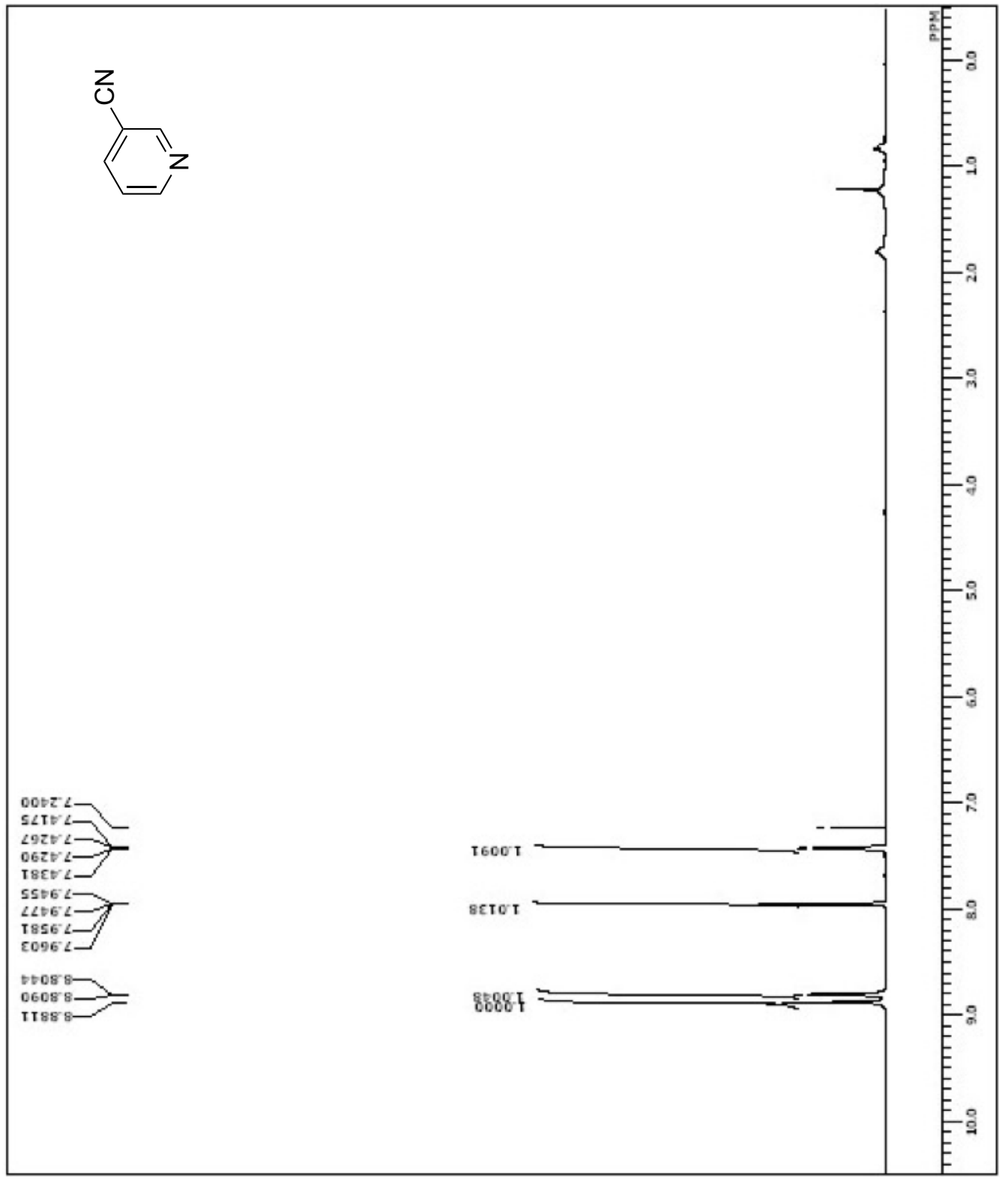


Nicotinonitrile (2m, $\left.\mathrm{CDCl}_{3}, 150 \mathrm{MHz}\right)$

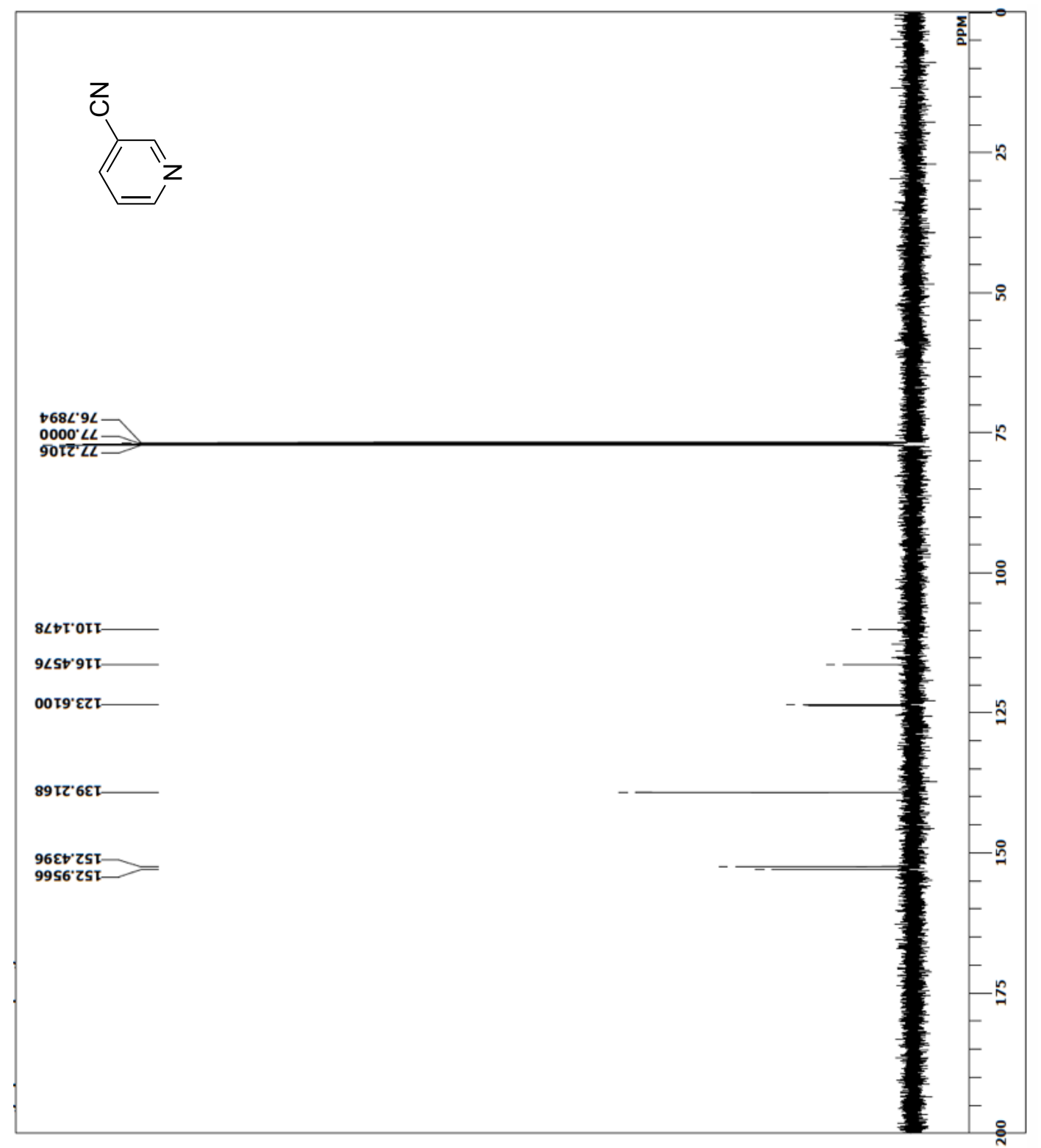

